\title{
MULTI-WAVELENGTH GOALS OBSERVATIONS OF STAR FORMATION AND ACTIVE GALACTIC NUCLEUS ACTIVITY IN THE LUMINOUS INFRARED GALAXY IC 883
}

\author{
F. Modica ${ }^{1}$, T. Vavilkin ${ }^{1,9}$, A. S. Evans ${ }^{1,2,3,9,10}$, D.-C. Kim ${ }^{2,3}$, J. M. Mazzarella ${ }^{4}$, K. Iwasawa ${ }^{5}$, A. Petric ${ }^{6}$, J. H. Howell ${ }^{6}$, \\ J. A. Surace ${ }^{6}$, L. Armus 6 , H. W. W. Spoon ${ }^{7}$, D. B. Sanders ${ }^{8}$, A. Wong ${ }^{3}$, And J. E. Barnes ${ }^{8}$ \\ ${ }^{1}$ Department of Physics \& Astronomy, Stony Brook University, Stony Brook, NY 11794-3800, USA; tvavilk@ vulcan.ess.sunysb.edu, aevans@ virginia.edu \\ ${ }^{2}$ National Radio Astronomy Observatory, 520 Edgemont Road, Charlottesville, VA 22903, USA \\ ${ }^{3}$ Department of Astronomy, University of Virginia, 530 McCormick Road, Charlottesville, VA 22904, USA; dk3wc@ virginia.edu, aww2b@ virginia.edu \\ ${ }^{4}$ Infrared Processing and Analysis Center, California Institute of Technology, MS 100-22, Pasadena, CA 91125, USA; mazz@ipac.caltech.edu \\ ${ }^{5}$ ICREA and Institut de Ciències del Cosmos (ICC), Universitat de Barcelona (IEEC-UB), Martí i Franquès 1, 08028 Barcelona, Spain; kazushi.iwasawa@ icc.ub.edu \\ ${ }^{6}$ Spitzer Science Center, Pasadena, CA 91125, USA; jhhowell@ ipac.caltech.edu, jason@ipac.caltech.edu, lee@ ipac.caltech.edu \\ ${ }^{7}$ Department of Astronomy, Cornell University, Ithaca, NY 14853, USA; spoon@isc.astro.cornell.edu \\ ${ }^{8}$ Institute of Astronomy, University of Hawaii, 2680 Woodlawn Dr., Honolulu, HI 96822, USA; sanders@ifa.hawaii.edu, barnes@ifa.hawaii.edu \\ Received 2010 August 17; accepted 2011 September 26; published 2011 December 9
}

\begin{abstract}
New optical Hubble Space Telescope (HST), Spitzer Space Telescope, Galaxy Evolution Explorer, and Chandra observations of the single-nucleus, luminous infrared galaxy (LIRG) merger IC 883 are presented. The galaxy is a member of the Great Observatories All-sky LIRG Survey and is of particular interest for a detailed examination of a luminous late-stage merger due to the richness of the optically visible star clusters and the extended nature of the nuclear X-ray, mid-IR, CO, and radio emission. In the HST Advanced Camera for Surveys images, the galaxy is shown to contain 156 optically visible star clusters distributed throughout the nuclear regions and tidal tails of the merger, with a majority of visible clusters residing in an arc $\sim 3-7 \mathrm{kpc}$ from the position of the mid-infrared core of the galaxy. The luminosity functions of the clusters have an $\alpha_{\mathrm{F} 435 \mathrm{~W}} \sim-2.17 \pm 0.22$ and $\alpha_{\mathrm{F} 814 \mathrm{~W}} \sim-2.01 \pm 0.21$, compared with $V$-band-derived values measured for the well-studied LIRG NGC 34 and the Antennae Galaxy of $\alpha \sim-1.7 \pm 0.1$ and $-2.13 \pm 0.07$, respectively. Further, the colors and absolute magnitudes of the majority of the clusters are consistent with instantaneous burst population synthesis model ages in the range of a few $\times 10^{7}-10^{8} \mathrm{yr}$ (for $10^{5} M_{\odot}$ clusters), but may be as low as few $\times 10^{6} \mathrm{yr}$ with extinction factored in. The X-ray and mid-IR spectroscopies are indicative of predominantly starburst-produced nuclear emission, and the star formation rate, estimated based on the assumption that the radio and far-infrared luminosities are tracing the starburst population, is $\sim 80 M_{\odot} \mathrm{yr}^{-1}$. The kinematics of the $\mathrm{CO}$ emission and the morphology of both the CO and radio emission are consistent with the nuclear starburst being situated in a highly inclined disk $2 \mathrm{kpc}$ in diameter with an infrared surface brightness $\mu_{\mathrm{IR}} \sim 2 \times 10^{11} L_{\odot} \mathrm{kpc}^{-2}$, a factor of 10 less than that of the Orion star-forming region. Finally, the detection of the [Ne v] $14.32 \mu \mathrm{m}$ emission line is evidence that an active galactic nucleus (AGN) is present. The faintness of the line (i.e., [ $\mathrm{Ne} \mathrm{v}] /[\mathrm{Ne}$ II $] 12.8 \mu \mathrm{m} \sim 0.01$ ) and the small equivalent width of the $6.2 \mu \mathrm{m}$ polycyclic aromatic hydrocarbon feature $(=0.39 \mu \mathrm{m})$ are both indicative of a relatively weak AGN.
\end{abstract}

Key words: galaxies: active - galaxies: individual (IC 883) - galaxies: interactions

\section{INTRODUCTION}

The $z=0.0233$ galaxy IC 883 (= UGC 8387, Arp 193, B2 $\left.1318+343 ; L_{\mathrm{IR}}[8-1000 \mu \mathrm{m}] \sim 5.4 \times 10^{11} L_{\odot}\right)$ is a member of the luminous infrared galaxy (LIRG) class $\left(L_{\mathrm{IR}} \geqslant 10^{11} L_{\odot}\right.$; e.g., Sanders \& Mirabel 1996). As is the case with some of the local LIRG population, the high $L_{\mathrm{IR}}$ of IC 883 is the result of a recent galaxy-galaxy merger event-the galaxy is observed optically to be a single-nucleus, advanced merger with two tidal tails. It is optically classified as a Low Ionization Nuclear Emission Region galaxy (Kim et al. 1995; Veilleux et al. 1995), with the evidence thus far supporting star formation as its primary energy source. Millimeter (CO) observations of the galaxy show it to have $\sim 4 \times 10^{9} M_{\odot}$ of molecular gas (adopting $M\left[\mathrm{H}_{2}\right] / L_{\mathrm{CO}}^{\prime}=0.8 M_{\odot}\left(\mathrm{K} \mathrm{km} \mathrm{s}^{-1} \mathrm{Mpc}^{2}\right)^{-1}$; Downes \& Solomon 1998) within the inner several kiloparsecs; the morphology and velocity profile of the gas is consistent with it being situated in a highly inclined, rotating disk (Downes \&

\footnotetext{
9 Visiting Astronomer at the Infrared Processing and Analysis Center, California Institute of Technology, MS 100-22, Pasadena, CA 91125, USA.

${ }^{10}$ Visiting Astronomer at the Institute of Astronomy and Astrophysics, Academia Sinica, P.O. Box 23-141, Taipei 106, Taiwan.
}

Solomon 1998; Bryant \& Scoville 1999). Furthemore, the HST NICMOS (Scoville et al. 2000) and Keck mid-IR (Soifer et al. 2001) imaging data also show evidence of a bright, edge-on disk $\sim 4^{\prime \prime}(2 \mathrm{kpc})$ in length in the nuclear region of the merger.

In this paper, new Hubble Space Telescope (HST), Spitzer Space Telescope, Chandra X-Ray Observatory, Galaxy Evolution Explorer $(G A L E X)$, and Owens Valley Millimeter Array (OVRO) observations, and archival Very Large Array (VLA) and HST NICMOS data, of IC 883 are presented. The galaxy is a member of the Great Observatories All-sky LIRG Survey (GOALS; Armus et al. 2009), which is a multi-wavelength survey of a complete sample of LIRGs in the IRAS Revised Bright Galaxy Sample (RBGS; Sanders et al. 2003); the present paper is the third in a series focusing on a single merger system. In the particular case of IC 883, the presence in a late-stage merger of $>100$ optically visible star clusters, combined with the spatial extent of the nuclear X-ray, mid-IR, CO, and radio emission, makes it ideally suited for examining the properties of distributed optically visible star formation and for constraining the nuclear activity in IC 883.

This paper is divided into six sections. In Section 2, the observations and data reduction are summarized. Section 3 contains a 
general overview of the results, followed by a discussion of the cluster identification and photometric methods in Section 4 (for a more detailed description of cluster identification and photometry, see T. Vavilkin et al. 2012, in preparation). Section 5 covers two major topics. First, the optical properties (distribution, luminosity function, and ages) of the $H S T$-visible star clusters are derived. Second, the discussion is expanded to include the broader GOALS data set, thus placing the optical observations, and the observed activity in IC 883, into the proper context. A summary of the findings (Section 6) concludes the paper.

The galaxy IC 883 has a heliocentric radial velocity of $6985( \pm 3) \mathrm{km} \mathrm{s}^{-1}$ (Rothberg \& Joseph 2006). The velocity, corrected for perturbations by the Virgo Cluster, the Great Attractor, and the Shapley Supercluster (Mould et al. 2000), is $7000( \pm 15) \mathrm{km} \mathrm{s}^{-1}$. Correcting the corresponding $z$ for Hubble flow in a cosmology with $H_{0}=70 \mathrm{~km} \mathrm{~s}^{-1} \mathrm{Mpc}^{-1}, \Omega_{\mathrm{m}}=0.28$, and $\Omega_{\Lambda}=0.72$ (Hinshaw et al. 2009) results in a luminosity distance to IC 883 of $110 \mathrm{Mpc}$ and a scale of $0.507 \mathrm{kpc} /{ }^{\prime \prime}$ (see also Armus et al. 2009).

\section{OBSERVATIONS AND DATA REDUCTION}

HST observations of IC 883 with the Wide Field Channel (WFC) of the Advanced Camera for Surveys (ACS) were obtained on 2006 January 11 as part of a much larger campaign (HST-GOALS; A. S. Evans et al. 2012, in preparation) to image a complete sample of $88 L_{\mathrm{IR}} \geqslant 10^{11.4} L_{\odot}$ IRAS galaxies in the RBGS (Sanders et al. 2003). The galaxy was imaged with the $F 435 W$ and $F 814 W$ filters in a single orbit, with integration times of 1290 and $740 \mathrm{~s}$, respectively. The data were reduced in the manner described in A. S. Evans et al. (2012, in preparation).

The Spitzer Infrared Array Camera (IRAC) and Multiband Imaging Photometer (MIPS) observations were obtained on 2005 June 11 and June 20, respectively, as part of the Spitzer component of GOALS (J. M. Mazzarella et al. 2012, in preparation). Integration times correspond to $150 \mathrm{~s}$ for the IRAC $3.6 \mu \mathrm{m}, 4.5 \mu \mathrm{m}, 5.8 \mu \mathrm{m}$, and $8 \mu \mathrm{m}$ channels and 48,38 , and $25 \mathrm{~s}$ for the MIPS $24 \mu \mathrm{m}, 70 \mu \mathrm{m}$, and $160 \mu \mathrm{m}$ channels, respectively. The Spitzer data were reduced in the manner described in detail in J. M. Mazzarella et al. (2012, in preparation).

The high-resolution Spitzer Infrared Spectrograph (IRS) data on IC 883 was taken as part of a Cycle 2 Spitzer observing campaign (PID 20549 AOR key 1483926). The source was observed with the high-resolution modules $(120 \mathrm{~s} \times 1$ cycles in $\mathrm{SH}$ and $60 \mathrm{~s} \times 1$ cycles in $\mathrm{LH})$. No offsets were taken in this program. The low-resolution SL data $(120 \mathrm{~s} \times 2$ cycles $)$ were taken as part of the Spitzer observing campaign (PID 3237 AOR key 10509824). The starting point for the data reduction was the Basic Calibrated Data processed by the Spitzer Science Center (SSC) pipeline (version S15.3.0). The SL and SH modules were first corrected for rogue pixels using an automatic search-and-interpolate algorithm (which was supplemented by visual examination). In order to estimate the background in the SL module, the two nods were subtracted. To extract onedimensional spectra, the Spectroscopic Modeling Analysis and Reduction Tool (Higdon et al. 2004) was used. The extraction apertures were tapered with wavelength to match the pointspread function for SL data and encompassed the entire slit for SH data. For SL, the two one-dimensional nod spectra for the orders SL1 and SL2 were combined separately and then the orders were stitched by trimming a few pixels from one or the other order. For SH, the two nods were combined and then stitched together by applying multiplicative offsets for each order that were linear in flux density versus wavelength (effectively removing a tilt artifact from certain orders where necessary). The zodiacal light in the $\mathrm{SH}$ spectra was subtracted using a blackbody fit to the Spitzer Planning Observations Tool zodiacal estimates at $10 \mu \mathrm{m}$ and $20 \mu \mathrm{m}$.

The GALEX far-UV $\left(\lambda_{\text {eff }}=0.1528 \mu \mathrm{m}\right)$ and near-UV $\left(\lambda_{\text {eff }}=\right.$ $0.2271 \mu \mathrm{m}$ ) imaging observations were done as part of the GALEX All-Sky Survey. The data were obtained on 2004 April 4 with an integration time of $119 \mathrm{~s}$ per band and were reduced using the standard GALEX pipeline.

The Chandra X-ray Observatory observations were obtained on 2007 February 19. The total integration time of the observations was $14.07 \mathrm{ks}$ and the data were reduced using the standard pipeline.

The $\mathrm{CO}(1 \rightarrow 0)$ observations of IC 883 were obtained during three observing periods from 1999 December to 2000 January with the Owens Valley Millimeter Array (OVRO). The array consists of six $10.4 \mathrm{~m}$ telescopes, and the longest observed baseline was $242 \mathrm{~m}$. Each baseline was configured with $120 \times 4 \mathrm{MHz}$ digital correlators. During the observations, the nearby quasar $1308+326$ was observed every 25 minutes to monitor phase and gain variations, and 3C 273 was observed to determine the passband structure. Finally, observations of Uranus were made for absolute flux calibration. The OVRO data were reduced and calibrated using the standard Owens Valley Millimeter Array program (MMA; Scoville et al. 1993). The data were then exported to the mapping program DIFMAP (Shepherd et al. 1995) for extraction of the intensity map and spectra.

The nuclear region of reduced images of IC 883 is shown in Figure 1 in order of increasing wavelength. Note that, due to the similarity in the morphology of IC 883 in the far- and near-UV, at 3.6 and $4.5 \mu \mathrm{m}$, and at 5.8 and $8.0 \mu \mathrm{m}$, only the near-UV, $3.6 \mu \mathrm{m}$, and $8.0 \mu \mathrm{m}$ images are shown. Figure 1 also includes an $8.4 \mathrm{GHz}$ radio image of IC 883 (Condon et al. 1991). Figure 2 contains the whole galaxy at the near-UV wavelength, in both $H S T$ filters, and at $3.6 \mu \mathrm{m}$.

\section{RESULTS}

The global appearance of the galaxy IC 883 is similar over the $0.1-3.6 \mu \mathrm{m}$ wavelength range (Figures 1 and 2), but its large-scale morphology is easiest to assess in the HST ACS images due to their depth and high resolution. The galaxy consists of a diffuse, elongated main body $17 \mathrm{kpc}$ in length with two tidal tails extending in the southeastern and southwestern directions (Figure 2(b) and (c)). The southeastern tail is linear in appearance with a projected full extent of $20 \mathrm{kpc}$ from the main body. In contrast, the southwestern tail extends from the main body out to a projected distance of $27 \mathrm{kpc}$, then back toward the main body where it eventually becomes difficult to distinguish against the high surface brightness haze. In addition to the large-scale structure visible in the HST images, a small fraction of the more than 100 bright knots (see Section 4), which are presumably luminous star clusters formed as a result of the merger, in IC 883 are shown in Figures 1(c) and (d) to be distributed within the extended nuclear region and main body of the galaxy.

Figure 1(b) shows the main body of IC 883 in the nearUV. The highest surface brightness emission in the near-UV emanates from the southern half of its main body and is offset from the bright nuclear cores visible in the Spitzer IRAC and MIPS images (Figures 1(e) and (f)). 


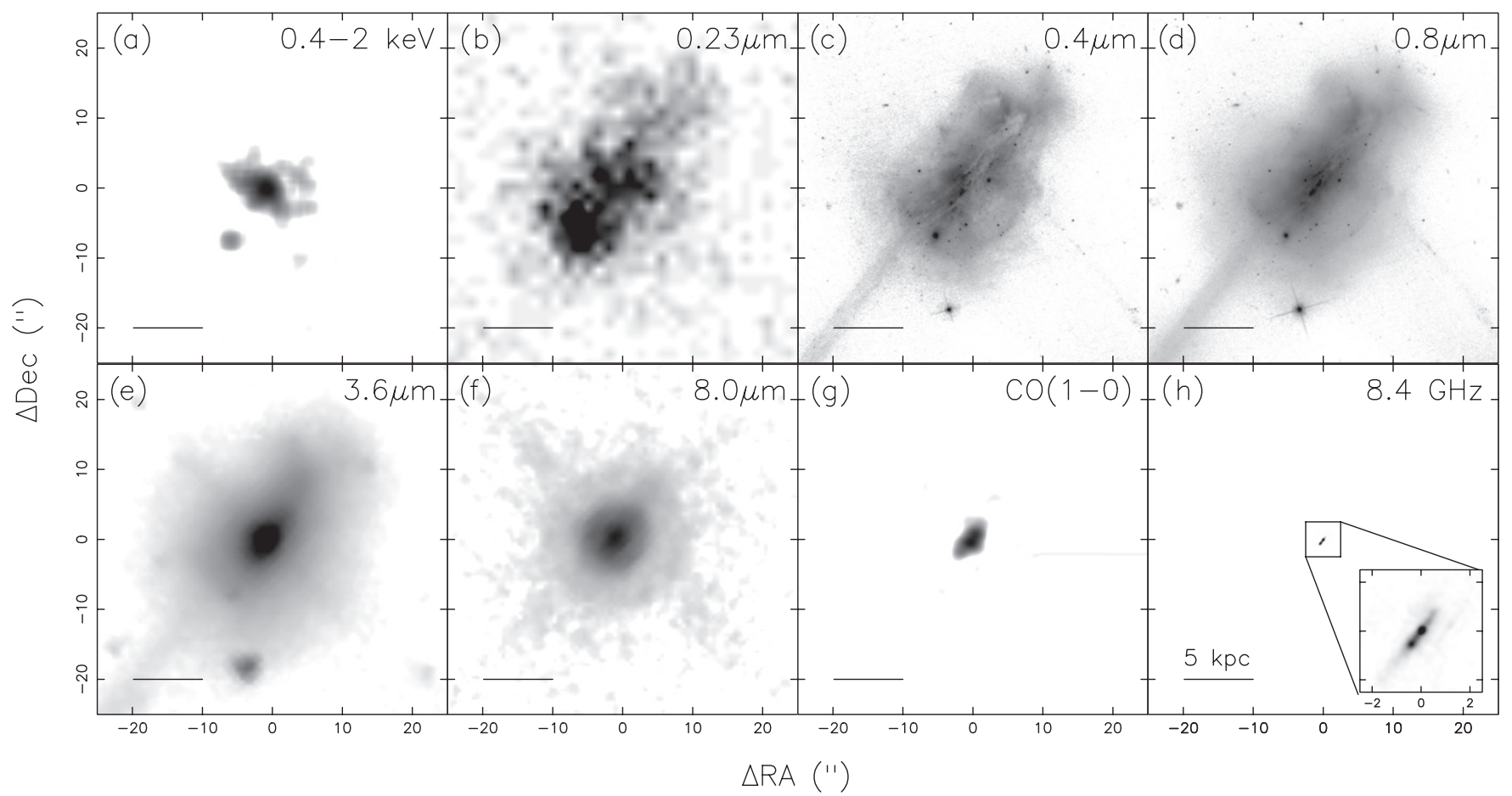

Figure 1. Multi-wavelength view of the nuclear region and main body of IC 883 displayed in order of increasing wavelength. The images have been spatially registered such that $\Delta$ R.A. $=0$ and $\Delta$ decl. $=0$ correspond to R.A. $=13: 20: 35.4$ and decl. $=+34: 08: 22.6$ (J2000). The images shown are as follows: (a) Chandra; (b) GALEX; (c), (d) HST; (e), (f) Spitzer; (g) OVRO; and (h) VLA. With the exception of the GALEX and VLA images, all of the images are shown with a logarithmic stretch. The integrated intensity CO map is shown with natural weighting with a beam FWHM of $1^{\prime \prime} .92 \times 11^{\prime \prime} .35$ and a position angle of 79.7. In all of the images, north is up and east is to the left.

At longer wavelengths, the inner few kiloparsecs of IC 883 make up the bulk of the emission. At $8 \mu \mathrm{m}$, the galaxy consists of an unresolved nucleus which is surrounded by a faint haze. In the lower resolution MIPS images (not shown), which cover the wavelength range $24-160 \mu \mathrm{m}$, the galaxy appears as a single, unresolved point source. The true compactness of the IR-emitting region may be best represented by the $\mathrm{CO}(1 \rightarrow 0)$ and $8.4 \mathrm{GHz}$ radio emission images (Figures $1(\mathrm{~g})$ and $(\mathrm{h})$; see discussion in Section 5.3). Both the $\mathrm{CO}$ and radio emission have elongated morphologies with full extents of $\sim 2 \mathrm{kpc}$. Two high surface brightness knots, separated by $350 \mathrm{pc}$, are observed in the radio image, one of which is spatially aligned with the $8 \mu \mathrm{m}$ core of IC 883.

A substantial amount of literature exists on the $\mathrm{CO}$ properties of IC 883. The measured CO flux density of the present data set is $132 \pm 12 \mathrm{Jy} \mathrm{km} \mathrm{s}^{-1}$, with an additional calibration uncertainty that is likely to be as high as $20 \%$. By comparison, Downes \& Solomon (1998) and Bryant \& Scoville (1999) report CO flux densities from their interferometric observations of $161 \pm$ $32 \mathrm{Jy} \mathrm{km} \mathrm{s}^{-1}$ and $202 \mathrm{Jy} \mathrm{km} \mathrm{s}^{-1}$, respectively. Morphologically, the appearance of the CO-synthesized intensity map shown in Figure $1(\mathrm{~g})$ is similar to those of Downes \& Solomon (1998) and Bryant \& Scoville (1999). There are also several published single-dish measurements of IC 883 (Mazzarella et al. 1993; Downes \& Solomon 1998; Evans et al. 2005); their reported CO flux densities are 220, 162, and $177 \mathrm{Jy} \mathrm{km} \mathrm{s}^{-1}$, respectively.

The X-ray emission is composed of extended emission centered on the $8 \mu \mathrm{m}$ galaxy core and a weak, point-like source, probably associated with a star cluster to the southeast (SE). The position of the primary extended emission centroid is $13^{\mathrm{h}} 20^{\mathrm{m}} 35^{\mathrm{s}} 3,+34^{\mathrm{d}} 08^{\mathrm{m}} 21^{\mathrm{s}} .9(\mathrm{~J} 2000)$ and of the SE compact source is $13^{\mathrm{h}} 20^{\mathrm{m}} 35^{\mathrm{s}} .7,+34^{\mathrm{d}} 08^{\mathrm{m}} 14^{\mathrm{s}} .3(\mathrm{~J} 2000)$.
The integrated counts of the primary X-ray component are $251.4 \pm 16.0$ counts in the $0.4-7 \mathrm{keV}$ band and $15.8 \pm 4.0$ counts for the SE component. The hardness ratio, defined as $\mathrm{HR}=(H-S) /(H+S)$, where $H$ is $2-8 \mathrm{keV}$ counts and $S$ is $0.5-2 \mathrm{keV}$ counts, of the two components are: $\mathrm{HR}$ (main) $=$ $-0.55 \pm 0.07$ and $\mathrm{HR}(\mathrm{SE})=-0.64 \pm 0.30$.

The soft X-ray emission (0.4-2 keV; see Figure 1(a)) is extended in the direction of northeast-southwest (SW), nearly perpendicular to the major axis of the galaxy. Very faint filamentary emission might extend up to $16^{\prime \prime}(8 \mathrm{kpc})$ to the $\mathrm{SW}$. The brightness distribution of the soft X-ray emission is rather flat: when fitted with a King profile, it is characterized by a large core radius of $10^{\prime \prime}(\sim 5 \mathrm{kpc})$ and a steep index of 10 (indicating a rapid decline beyond the core radius). The $2-7 \mathrm{keV}$ emission is marginally resolved.

The X-ray spectrum (Figure 3) was extracted only from the primary emission region (the SE component is too faint to construct a spectrum for an independent investigation). The spectrum consists of thermal emission in the soft band. There is a possible presence of a hard tail in the Chandra spectrum above $4 \mathrm{keV}$. Given the limited detected counts, no good constraint on the spectral shape can be obtained, but its slope could be rather flat, with a photon index $\Gamma \leqslant 1$. The soft X-ray emission features can be described by a thermal emission spectrum with a temperature of $k T=1.0 \pm 0.1 \mathrm{keV}$ with an $\alpha$-element-enhanced metallicity pattern. This is consistent with an interstellar medium enriched by core-collapse supernovae, as is expected from a starburst. The Si XIII is very strong with an equivalent width of $0.26 \mathrm{keV}$, which requires $\sim 3$ times more metallicity than the other $\alpha$-elements. Since the Si XIII line is on the higher energy side of the thermal spectrum, the enhancement could be due to an extra contribution of active galactic nucleus (AGN) photoionized gas, as suspected based 


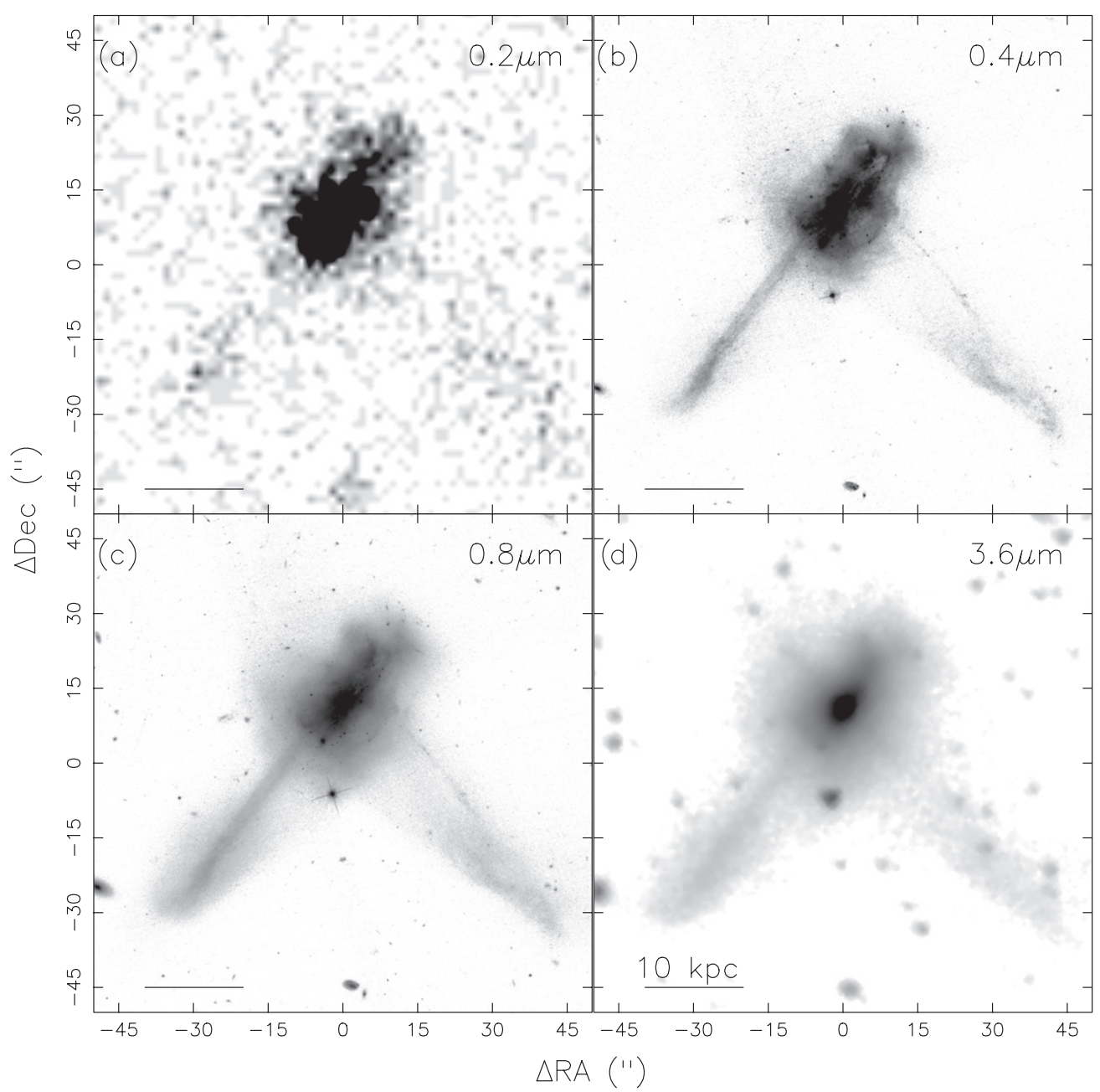

Figure 2. Multi-wavelength, wide-field view of IC 883 displayed in order of increasing wavelength. The images shown are as follows: (a) GALEX; (b), (c) HST; and (d) Spitzer. With the exception of the GALEX image, all of the images are shown with a logarithm stretch. In all of the images, north is up and east is to the left.

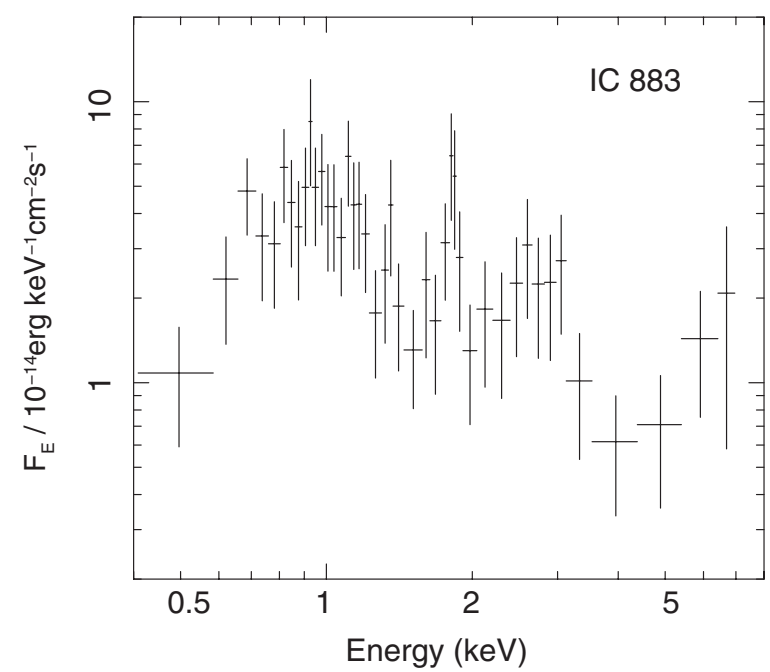

Figure 3. Response-corrected X-ray spectrum of IC 883 obtained from the Chandra ACIS-S. The flux density is plotted in units of $10^{-14} \mathrm{erg} \mathrm{s}^{-1} \mathrm{keV}^{-1}$ and the energy is in units of $\mathrm{keV}$ in the observer frame. The soft $\mathrm{X}$-ray emission can be modeled as thermal emission with a temperature of $k T \sim 0.7 \mathrm{keV}$. A strong Si XIII line at $1.8 \mathrm{keV}$ may suggest $\alpha$-element-enriched gas composition. The hard X-ray tail above $3 \mathrm{keV}$ comes from the central compact source and its spectral shape is not well constrained due to small detected counts.

on the similarly strong Si XIII line found in other GOALS galaxies hosting Compton thick AGNs (Iwasawa et al 2011). The soft
Table 1

Chandra X-ray Fluxes and Luminosities

\begin{tabular}{lcc}
\hline \hline $\begin{array}{l}\text { Band } \\
(\mathrm{keV})\end{array}$ & $\begin{array}{c}\text { Flux } \\
\left(\mathrm{erg} \mathrm{s}^{-1} \mathrm{~cm}^{-2}\right)\end{array}$ & $\begin{array}{c}\text { Luminosity } \\
\left(\mathrm{erg} \mathrm{s}^{-1}\right)\end{array}$ \\
\hline $\begin{array}{c}\text { Nuclear } \\
0.5-2\end{array}$ & $4.2 \pm 0.4 \times 10^{-14}$ & $6.1 \pm 0.6 \times 10^{40}$ \\
$2-7$ & $5.1 \pm 0.8 \times 10^{-14}$ & $7.4 \pm 1.2 \times 10^{40}$ \\
$2-10$ & $6.5 \pm 1.2 \times 10^{-14}$ & $9.4 \pm 1.2 \times 10^{40}$ \\
SE source & & \\
$0.5-2$ & $0.27 \pm 0.08 \times 10^{-14}$ & $0.39 \pm 0.11 \times 10^{40}$ \\
\hline
\end{tabular}

band spectrum may likely be best explained by a non-solar abundance ratio with enhanced $\alpha$-elements. A further investigation is reported by Iwasawa et al. (2011). The observed (i.e., uncorrected for absorption) fluxes and luminosities of the nuclear and SE source X-ray emission are summarized in Table 1.

The low+high-resolution composite Spitzer IRS spectrum (Figure 4) shows strong emission from forbidden lines, $\mathrm{H}_{2}$ emission, and polycyclic aromatic hydrocarbon (PAH). The [Ne II] $12.8 \mu \mathrm{m}$, [Ne III] $15.6 \mu \mathrm{m}, \mathrm{H}_{2} S(1)$, [S III] $33.48 \mu \mathrm{m}$, and [Si II] $34.82 \mu \mathrm{m}$ emission lines are the strongest non-PAH emission features in the spectrum. The fluxes of the detected forbidden and $\mathrm{H}_{2}$ emission lines are provided in Table 2. Further, the equivalent width of the $6.4 \mu \mathrm{m}$ PAH emission feature (Figure 4(a)) is measured to be $0.39 \mu \mathrm{m}$. 

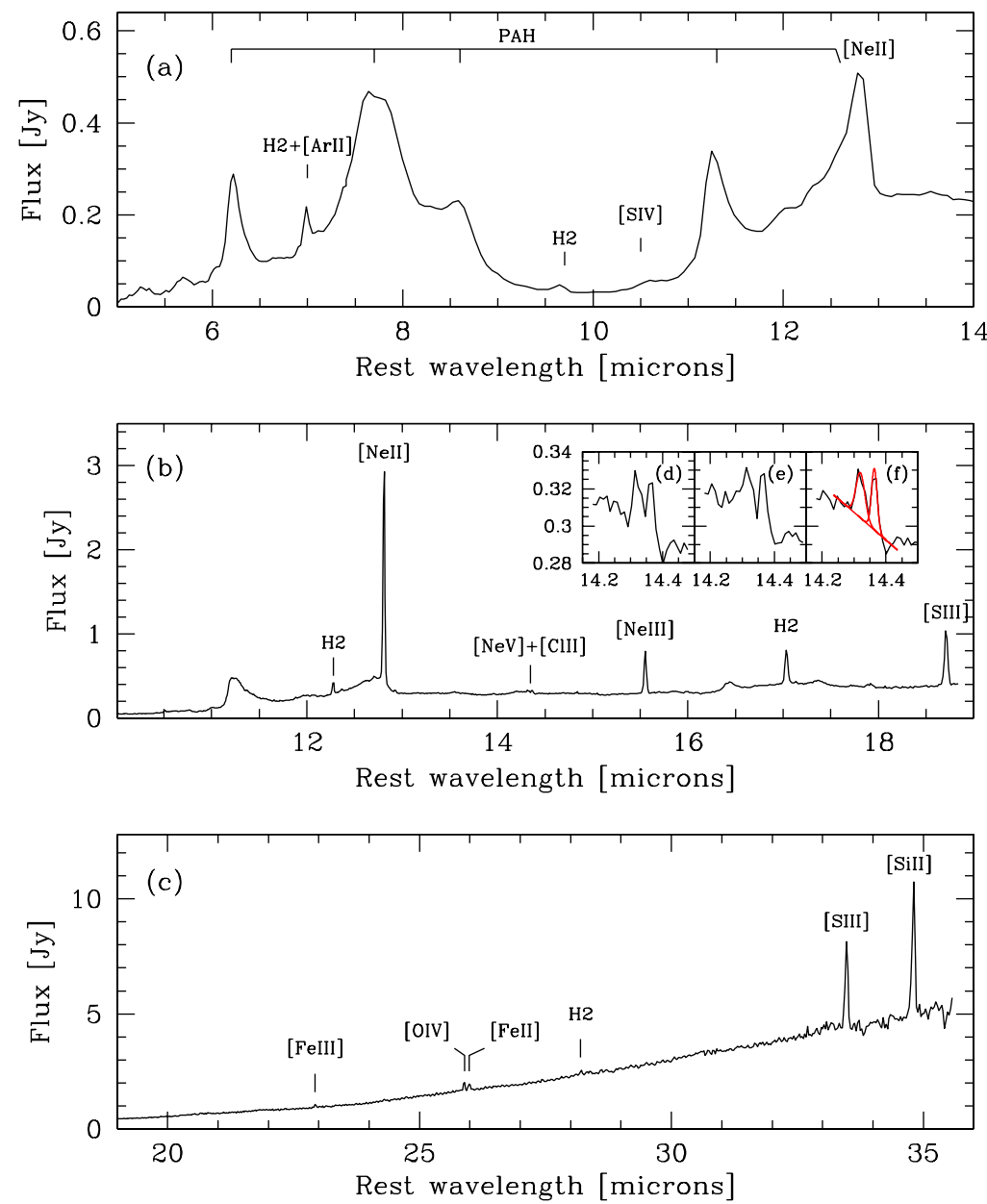

Figure 4. Low (a) and high ((b) and (c)) resolution Spitzer IRS spectrum of IC 883. The insets in (b), which are labeled (d), (e), and (f), are centered on the location of the [Ne v] $14.3 \mu \mathrm{m}$ and [Cl II] $14.4 \mu \mathrm{m}$ lines. Insets (d) and (e) are spectra from the two individual nods, and (f) is the average of the two nods. The simultaneous fits to the $[\mathrm{Ne} \mathrm{v}]$ and $[\mathrm{Cl}$ II] emission features are shown in red (f). The narrowness of the [Cl II] line is likely due to the trough observed in both nods at $14.4 \mu \mathrm{m}$.

Table 2

IRS Emission Lines and Line Flux Densities

\begin{tabular}{lr}
\hline \hline Emission Line & $\begin{array}{c}\text { Flux Density } \\
\left(\mathrm{W} \mathrm{cm}^{-2}\right)\end{array}$ \\
\hline $\mathrm{H}_{2} \mathrm{~S}(3) 9.66 \mu \mathrm{m}$ & $6.52 \pm 0.41 \times 10^{-21}$ \\
{$[\mathrm{~S} \mathrm{IV}] 10.51 \mu \mathrm{m}$} & $1.20 \pm 0.14 \times 10^{-21}$ \\
$\mathrm{H}_{2} \mathrm{~S}(2) 12.28 \mu \mathrm{m}$ & $6.15 \pm 0.32 \times 10^{-21}$ \\
$\mathrm{Hu}-\alpha 12.37 \mu \mathrm{m}$ & $1.04 \pm 0.11 \times 10^{-21}$ \\
{$[\mathrm{Ne}$ II $] 12.8 \mu \mathrm{m}$} & $1.13 \pm 0.012 \times 10^{-19}$ \\
{$[\mathrm{Ne}$ V] $14.32 \mu \mathrm{m}$} & $1.17 \pm 0.23 \times 10^{-21}$ \\
{$[\mathrm{Ne}$ III $] 15.55 \mu \mathrm{m}$} & $1.85 \pm 0.018 \times 10^{-20}$ \\
$\mathrm{H}_{2} \mathrm{~S}(1) 17.03 \mu \mathrm{m}$ & $1.48 \pm 0.037 \times 10^{-20}$ \\
{$[\mathrm{~S} \mathrm{III}] 18.71 \mu \mathrm{m}$} & $2.20 \pm 0.061 \times 10^{-20}$ \\
{$[\mathrm{Fe}$ III $] 22.92 \mu \mathrm{m}$} & $2.10 \pm 0.11 \times 10^{-21}$ \\
{$[\mathrm{O}$ IV $] 25.89 \mu \mathrm{m}$} & $6.53 \pm 0.072 \times 10^{-21}$ \\
{$[\mathrm{Fe}$ II $] 25.99 \mu \mathrm{m}$} & $5.53 \pm 0.67 \times 10^{-21}$ \\
$\mathrm{H}_{2} \mathrm{~S}(0) 28.22 \mu \mathrm{m}$ & $2.47 \pm 0.49 \times 10^{-21}$ \\
{$[\mathrm{~S} \mathrm{III}] 33.48 \mu \mathrm{m}$} & $6.41 \pm 0.39 \times 10^{-20}$ \\
{$[\mathrm{Si}$ II $] 34.82 \mu \mathrm{m}$} & $9.77 \pm 0.72 \times 10^{-20}$ \\
\hline
\end{tabular}

\section{STAR CLUSTER IDENTIFICATION AND PHOTOMETRY}

A major goal of the present paper is to perform a photometric analysis of the luminous star clusters visible in the HST images. Before an automated routine for cluster identification could be applied to the images, contamination from foreground stars and distant background galaxies in the images had to be minimized. To address this, a mask of IC 883 was made by first creating a $21 \times 21$ median-smoothed version of the $F 435 W$ and $F 814 W$ images. The effect of this filtering is to minimize structures with spatial extents significantly smaller than the filter size (i.e., faint stars and distant, background galaxies). The image was then boxcar smoothed $(40 \times 40)$. The result of the filtering and smoothing is an image with high valued pixels where the galaxy is located (i.e., where the median filtering would produce a pixel value greater than zero) and low valued pixels in the background regions (where the median filtering would negate pixel counts associated with distant, background galaxies and faint foreground stars). The backgrounds, containing low pixel values, were then set to zero, while the high pixels were set to one. Finally, pixels associated with any bright stars in the image were set to zero. The resultant mask, which outlines the galaxy to a surface brightness of $\sim 25 \mathrm{mag} /{ }^{\prime \prime 2}$, was then multiplied by the original reduced image of the galaxy to set the regions outside of the galaxy equal to zero. The main body of IC 883 in the masked $F 435 W$ image is shown in Figure 5(a).

Once the mask was applied, the clusters in IC 883 were identified using Source Extractor (Bertin \& Arnouts 1996). The identification of clusters and the extraction of photometry is complicated by the non-uniform surface brightness of the underlying galaxy. To estimate and subtract the underlying 


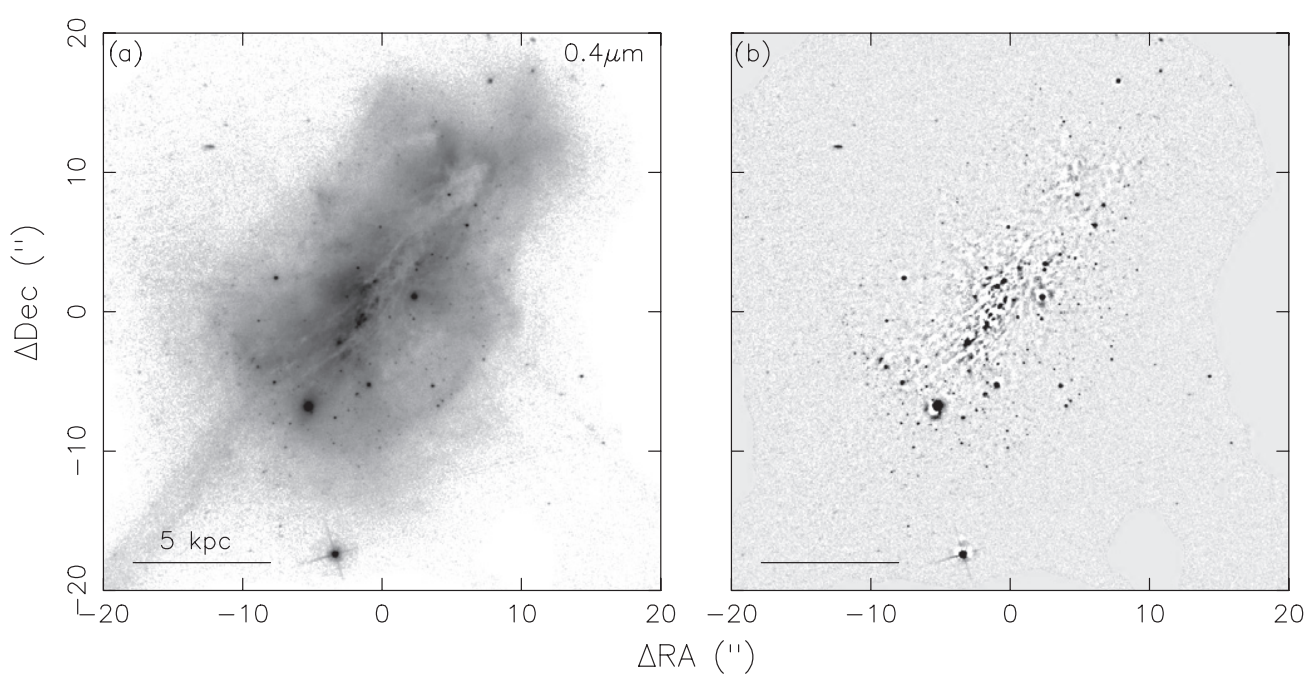

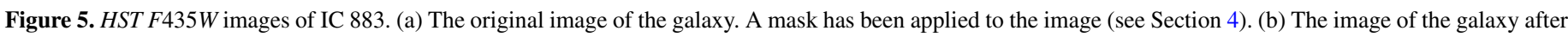

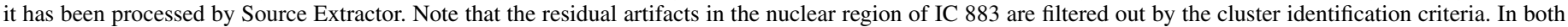
images, north is up and east is to the left.

galaxy, Source Extractor iteratively computes the median and standard deviation of the pixels within a mesh of $n \times n$ pixels; during each iteration, outlier pixels are discarded until all of the pixels within each mesh are within $\pm 3 \sigma$ of the median value. Several mesh sizes were tested; for each mesh, the photometry of several of the clusters was computed "by hand" via the image display and analysis program IPAC SKYVIEW (http://www.ipac.caltech.edu/skyview/) and compared to values estimated from the original image. The mesh size of $9 \times 9$ pixels did an efficient job of removing the galaxy and minimizing the creation of holes surrounding clusters during the extraction, and thus preserving the integrity of the cluster photometry. The $F 435 W$ image with the mesh applied is showed in Figure 5(b).

After Source Extractor was used to fit the underlying galaxy and to identify candidate clusters, the photometry of the candidates was computed using IDL routines. The photometry was calculated using the latest Vega magnitude zero points for the ACS/WFC $F 435 W$ and $F 814 W$ filters tabulated at http://www.stsci.edu/hst/acs/analysis/zeropoints. Photometric aperture corrections were applied to the 0.3 diameter aperture measurements using determinations of the curve of growth of bright, unresolved stars and clusters in the GOALS sample images; these curve of growth measurements are in agreement with values measured by Sirianni et al. (2005; Table 3). The final selection of clusters was based on the following criteria: (1) a detection at a signal-to-noise ratio $(\mathrm{S} / \mathrm{N}) \geqslant 5.0 \sigma$ in both the $F 435 W$ and $F 814 W$ images and (2) a full width at halfmaximum (FWHM) intensity in the range of $\sim 1.7-4.0$ pixels, which is consistent with the clusters being unresolved or partially resolved (4.0 pixels $\sim 100$ pc at the redshift of IC 883). Only cluster candidates that meet these criteria are used in the analysis that follows. Using this method, 156 star clusters are detected in IC 883.

The completeness limits were tested using the method described in detail in T. Vavilkin et al. (2012, in preparation). For the IC 883 images, the $F 435 W$ and $F 814 W$ images are found to be complete at the $50 \%$ level at the apparent magnitude $m_{\mathrm{F} 435 \mathrm{~W}} \sim 26.1$ (absolute magnitude of $M_{\mathrm{F} 435 \mathrm{~W}} \sim-9.15$ ) and $m_{\mathrm{F} 814 \mathrm{~W}} \sim 25.63\left(M_{\mathrm{F} 814 \mathrm{~W}} \sim-9.9\right)$ mag, respectively.

As stated above, the regions around the galaxy were masked before the clusters were identified, extracted, and analyzed. In order to estimate the contamination in each image by foreground stars and distant galaxies, these same procedures were also run on galaxy masked, "sky-only" versions of the ACS images. The ratio of the area of the image subtended by the galaxy relative to the area subtended by "sky-only" images provides a normalization to the sky-only source counts as a function of magnitude, and thus a measure of source contamination toward the galaxy.

\section{DISCUSSION}

\subsection{Properties of the Optically Visible Star Clusters}

One hundred fifty-six optical star clusters have been detected in the nuclear region, main body, and the extended tidal tails of IC 883. At the resolution of the $F 435 W$ images, the best constraint on the sizes of the unresolved star clusters is less than $\sim 57 \mathrm{pc}$ in diameter. Thus, regardless of the high-resolution nature of the images, the resolution is significantly poorer than the median effective diameter of Milky Way globular clusters ( $D_{\text {eff }} \sim 6$ pc; Van den Bergh 1996) and star clusters observed in the Antennae Galaxy ( $D_{\text {eff }} \sim 8 \pm 2$ pc; Whitmore et al. 1999).

Figure 6 is a histogram plot of the logarithm of the number of star clusters versus the cluster absolute magnitude, $\log (N)$ versus $M$, as measured in both the $F 435 W$ and $F 814 W$ images. Both the raw histogram and the histogram corrected for background contamination and completeness are shown, with the bulk of the clusters observed to have $M_{\mathrm{F} 814 \mathrm{~W}} \sim-13$ to -9 mag. A common parameter derived from such a plot is the power-law slope, $\alpha$, of the cluster luminosity function, i.e., the cluster luminosity function, $\psi(L)$, expressed in terms of luminosity, $L$, is of the form

$$
d N=\psi(L) d L \propto L^{\alpha} d L
$$

where $d N$ is the number of clusters between $L$ and $L+d L$. The best-fit luminosity function over the $M_{\mathrm{F} 814 \mathrm{~W}} \sim-13$ to -10 magnitude range (i.e., down to a completeness level of 50\%) has an $\alpha_{\mathrm{F} 814 \mathrm{~W}}=-2.01 \pm 0.21$, where $\alpha$ is related to the slope, $a(=d \log N / d M)$, of the histogram plotted in Figure 6 via

$$
\alpha=-(2 a+1) \text {. }
$$

For the $F 435 W$ data, the best-fit function over $M_{\mathrm{F} 435 \mathrm{~W}} \sim-12$ to -9 mag is $\alpha_{\mathrm{F} 435 \mathrm{~W}}=-2.17 \pm 0.22$. While a direct comparison 
Table 3

IC 883 Star Cluster Photometry

\begin{tabular}{|c|c|c|c|c|c|c|c|c|c|c|}
\hline \multirow[b]{2}{*}{$\begin{array}{l}\text { R.A. } \\
\left(\prime^{\prime \prime}\right)\end{array}$} & \multirow[b]{2}{*}{$\begin{array}{l}\text { Decl. } \\
\left({ }^{\prime \prime \prime}\right)\end{array}$} & \multirow[b]{2}{*}{$\begin{array}{c}\Delta D^{\mathrm{a}} \\
(\operatorname{arcsec})\end{array}$} & \multirow[b]{2}{*}{$\begin{array}{l}\Delta D^{\mathrm{a}} \\
(\mathrm{pc})\end{array}$} & \multicolumn{3}{|c|}{$F 435 W$} & \multicolumn{2}{|c|}{$F 814 W$} & \multicolumn{2}{|l|}{ Colors } \\
\hline & & & & $\begin{array}{c}m_{\mathrm{F} 435 \mathrm{~W}} \\
(\mathrm{mag})\end{array}$ & $\begin{array}{c}\text { err } \\
(\mathrm{mag})\end{array}$ & $\begin{array}{c}M_{\mathrm{F} 435 \mathrm{~W}} \\
(\mathrm{mag})\end{array}$ & $\begin{array}{c}m_{\mathrm{F} 814 \mathrm{~W}} \\
(\mathrm{mag})\end{array}$ & $\begin{array}{c}\text { err } \\
(\mathrm{mag})\end{array}$ & $\begin{array}{c}m_{\mathrm{F} 435 \mathrm{~W}}-m_{\mathrm{F} 814 \mathrm{~W}} \\
(\mathrm{mag})\end{array}$ & $\begin{array}{c}\text { err } \\
(\mathrm{mag})\end{array}$ \\
\hline 2035.699 & 814.68 & 8.71 & 4.41 & 18.99 & 0.00 & -16.27 & 18.21 & 0.00 & 0.75 & 0.00 \\
\hline 2035.094 & 822.51 & 3.39 & 1.72 & 19.95 & 0.00 & -15.31 & 19.17 & 0.00 & 0.75 & 0.00 \\
\hline 2035.889 & 823.87 & 6.69 & 3.39 & 21.66 & 0.00 & -13.60 & 20.91 & 0.00 & 0.72 & 0.00 \\
\hline 2035.352 & 816.22 & 6.10 & 3.09 & 21.77 & 0.00 & -13.49 & 21.04 & 0.00 & 0.70 & 0.00 \\
\hline 2034.792 & 827.58 & 8.91 & 4.52 & 22.46 & 0.00 & -12.80 & 21.32 & 0.00 & 1.11 & 0.00 \\
\hline 2035.412 & 820.57 & 1.82 & 0.92 & 22.72 & 0.03 & -12.54 & 19.47 & 0.01 & 3.22 & 0.03 \\
\hline 2035.896 & 816.42 & 8.87 & 4.49 & 22.78 & 0.01 & -12.48 & 21.83 & 0.01 & 0.92 & 0.01 \\
\hline 2034.986 & 816.14 & 7.76 & 3.93 & 22.91 & 0.00 & -12.35 & 22.24 & 0.01 & 0.64 & 0.01 \\
\hline 2034.897 & 829.77 & 9.55 & 4.84 & 23.05 & 0.01 & -12.21 & 22.23 & 0.02 & 0.79 & 0.02 \\
\hline 2035.418 & 824.55 & 2.36 & 1.20 & 23.14 & 0.01 & -12.12 & 21.10 & 0.01 & 2.01 & 0.01 \\
\hline 2035.545 & 813.87 & 8.76 & 4.44 & 23.42 & 0.01 & -11.84 & 22.49 & 0.01 & 0.90 & 0.01 \\
\hline 2035.420 & 815.56 & 6.78 & 3.44 & 23.50 & 0.01 & -11.76 & 21.76 & 0.01 & 1.71 & 0.01 \\
\hline 2034.661 & 837.89 & 17.98 & 9.11 & 23.62 & 0.01 & -11.64 & 22.71 & 0.01 & 0.88 & 0.01 \\
\hline 2034.744 & 829.04 & 10.31 & 5.23 & 23.70 & 0.01 & -11.56 & 22.12 & 0.01 & 1.55 & 0.01 \\
\hline 2034.127 & 816.79 & 16.31 & 8.27 & 23.72 & 0.01 & -11.54 & 22.75 & 0.01 & 0.94 & 0.01 \\
\hline 2035.081 & 824.43 & 4.16 & 2.11 & 23.90 & 0.03 & -11.36 & 21.69 & 0.01 & 2.18 & 0.03 \\
\hline 2035.481 & 822.51 & 1.45 & 0.73 & 23.90 & 0.03 & -11.36 & 19.87 & 0.00 & 4.00 & 0.03 \\
\hline 2035.991 & 817.49 & 9.16 & 4.65 & 23.92 & 0.02 & -11.34 & 23.32 & 0.03 & 0.57 & 0.04 \\
\hline 2035.291 & 827.48 & 5.30 & 2.69 & 24.04 & 0.02 & -11.22 & 23.19 & 0.02 & 0.82 & 0.03 \\
\hline 2034.954 & 814.71 & 9.13 & 4.63 & 24.05 & 0.01 & -11.21 & 23.27 & 0.02 & 0.75 & 0.02 \\
\hline 2035.514 & 817.20 & 5.44 & 2.76 & 24.05 & 0.02 & -11.21 & 22.90 & 0.02 & 1.12 & 0.03 \\
\hline 2034.992 & 825.52 & 5.68 & 2.88 & 24.05 & 0.03 & -11.21 & 21.92 & 0.01 & 2.10 & 0.03 \\
\hline 2035.988 & 820.81 & 7.88 & 3.99 & 24.08 & 0.01 & -11.18 & 23.33 & 0.02 & 0.72 & 0.02 \\
\hline 2035.096 & 820.93 & 3.61 & 1.83 & 24.12 & 0.02 & -11.14 & 23.45 & 0.03 & 0.64 & 0.04 \\
\hline 2035.235 & 821.06 & 2.04 & 1.03 & 24.37 & 0.03 & -10.89 & 23.35 & 0.08 & 0.99 & 0.09 \\
\hline 2035.803 & 813.45 & 10.44 & 5.29 & 24.43 & 0.02 & -10.83 & 23.64 & 0.02 & 0.76 & 0.03 \\
\hline 2036.016 & 818.08 & 9.14 & 4.63 & 24.65 & 0.04 & -10.61 & 23.94 & 0.04 & 0.68 & 0.06 \\
\hline 2034.420 & 838.62 & 20.23 & 10.26 & 24.68 & 0.02 & -10.58 & 24.04 & 0.02 & 0.61 & 0.03 \\
\hline 2035.757 & 816.12 & 7.90 & 4.00 & 24.84 & 0.06 & -10.42 & 24.17 & 0.07 & 0.64 & 0.09 \\
\hline 2035.553 & 812.04 & 10.55 & 5.35 & 24.93 & 0.03 & -10.33 & 24.34 & 0.04 & 0.56 & 0.05 \\
\hline 2035.469 & 814.39 & 8.03 & 4.07 & 24.94 & 0.03 & -10.32 & 22.57 & 0.01 & 2.34 & 0.03 \\
\hline 2034.691 & 818.01 & 9.38 & 4.76 & 24.95 & 0.02 & -10.31 & 24.25 & 0.03 & 0.67 & 0.04 \\
\hline 2035.347 & 817.70 & 4.62 & 2.34 & 24.98 & 0.06 & -10.28 & 23.93 & 0.05 & 1.02 & 0.08 \\
\hline 2035.291 & 812.92 & 9.43 & 4.78 & 24.99 & 0.04 & -10.27 & 24.24 & 0.03 & 0.72 & 0.05 \\
\hline 2035.829 & 811.78 & 12.05 & 6.11 & 25.01 & 0.03 & -10.25 & 24.31 & 0.04 & 0.67 & 0.05 \\
\hline 2034.975 & 835.00 & 13.67 & 6.93 & 25.05 & 0.04 & -10.21 & 23.97 & 0.04 & 1.05 & 0.06 \\
\hline 2035.424 & 818.94 & 3.44 & 1.74 & 25.07 & 0.09 & -10.19 & 24.67 & 0.30 & 0.37 & 0.31 \\
\hline 2034.906 & 825.24 & 6.47 & 3.28 & 25.08 & 0.06 & -10.18 & 24.47 & 0.07 & 0.58 & 0.09 \\
\hline 2035.406 & 816.37 & 5.97 & 3.03 & 25.09 & 0.05 & -10.17 & 24.72 & 0.10 & 0.34 & 0.11 \\
\hline 2035.900 & 813.49 & 11.07 & 5.61 & 25.09 & 0.04 & -10.17 & 24.49 & 0.05 & 0.57 & 0.06 \\
\hline 2035.116 & 821.21 & 3.28 & 1.66 & 25.12 & 0.06 & -10.14 & 24.40 & 0.08 & 0.69 & 0.10 \\
\hline 2035.760 & 817.51 & 6.87 & 3.48 & 25.15 & 0.09 & -10.11 & 24.26 & 0.07 & 0.86 & 0.11 \\
\hline 2035.213 & 823.85 & 2.47 & 1.25 & 25.24 & 0.09 & -10.02 & 22.74 & 0.08 & 2.47 & 0.12 \\
\hline 2033.409 & 84.12 & 30.27 & 15.35 & 25.26 & 0.03 & -10.00 & 24.89 & 0.05 & 0.34 & 0.06 \\
\hline 2035.722 & 820.81 & 4.67 & 2.37 & 25.28 & 0.06 & -9.98 & 24.13 & 0.06 & 1.12 & 0.09 \\
\hline 2035.602 & 815.60 & 7.32 & 3.71 & 25.29 & 0.08 & -9.97 & 24.90 & 0.12 & 0.36 & 0.14 \\
\hline 2035.197 & 817.79 & 4.97 & 2.52 & 25.31 & 0.05 & -9.95 & 23.46 & 0.02 & 1.82 & 0.05 \\
\hline 2034.695 & 818.95 & 8.97 & 4.55 & 25.37 & 0.04 & -9.89 & 24.65 & 0.05 & 0.69 & 0.06 \\
\hline 2034.886 & 821.97 & 5.92 & 3.00 & 25.40 & 0.05 & -9.86 & 24.97 & 0.10 & 0.40 & 0.11 \\
\hline 2035.287 & 811.72 & 10.65 & 5.40 & 25.41 & 0.05 & -9.85 & 24.65 & 0.05 & 0.73 & 0.07 \\
\hline 2035.954 & 810.00 & 14.37 & 7.29 & 25.46 & 0.05 & -9.80 & 24.97 & 0.08 & 0.46 & 0.09 \\
\hline 2034.617 & 828.10 & 11.01 & 5.58 & 25.47 & 0.05 & -9.79 & 24.45 & 0.04 & 0.99 & 0.06 \\
\hline 2034.786 & 824.83 & 7.67 & 3.89 & 25.50 & 0.06 & -9.76 & 24.70 & 0.06 & 0.77 & 0.09 \\
\hline 2035.699 & 813.03 & 10.21 & 5.17 & 25.50 & 0.05 & -9.76 & 24.96 & 0.08 & 0.51 & 0.09 \\
\hline 2034.000 & 812.03 & 19.78 & 10.03 & 25.50 & 0.04 & -9.76 & 24.52 & 0.03 & 0.95 & 0.05 \\
\hline 2035.177 & 818.72 & 4.26 & 2.16 & 25.55 & 0.06 & -9.71 & 24.69 & 0.08 & 0.83 & 0.10 \\
\hline 2034.994 & 821.96 & 4.64 & 2.35 & 25.57 & 0.07 & -9.69 & 25.23 & 0.15 & 0.31 & 0.17 \\
\hline 2036.169 & 818.49 & 10.70 & 5.42 & 25.59 & 0.06 & -9.67 & 24.47 & 0.06 & 1.09 & 0.09 \\
\hline 2035.005 & 830.62 & 9.52 & 4.83 & 25.60 & 0.10 & -9.66 & 24.98 & 0.16 & 0.59 & 0.19 \\
\hline 2034.938 & 833.94 & 12.88 & 6.53 & 25.60 & 0.08 & -9.66 & 24.89 & 0.08 & 0.68 & 0.11 \\
\hline 2035.627 & 817.31 & 5.97 & 3.03 & 25.61 & 0.11 & -9.65 & 24.94 & 0.17 & 0.64 & 0.20 \\
\hline 2035.669 & 827.78 & 6.65 & 3.37 & 25.62 & 0.05 & -9.64 & 25.01 & 0.08 & 0.58 & 0.09 \\
\hline 2035.430 & 825.27 & 3.09 & 1.57 & 25.64 & 0.08 & -9.62 & 21.82 & 0.01 & 3.79 & 0.08 \\
\hline 2035.092 & 830.03 & 8.51 & 4.32 & 25.65 & 0.08 & -9.61 & 25.00 & 0.14 & 0.62 & 0.16 \\
\hline
\end{tabular}


Table 3

(Continued)

\begin{tabular}{|c|c|c|c|c|c|c|c|c|c|c|}
\hline \multirow[b]{2}{*}{$\begin{array}{l}\text { R.A. } \\
\left({ }^{\prime \prime \prime}\right)\end{array}$} & \multirow[b]{2}{*}{$\begin{array}{l}\text { Decl. } \\
\left({ }^{\prime \prime}\right)\end{array}$} & \multirow[b]{2}{*}{$\begin{array}{c}\Delta D^{\mathrm{a}} \\
(\operatorname{arcsec})\end{array}$} & \multirow[b]{2}{*}{$\begin{array}{l}\Delta D^{\mathrm{a}} \\
(\mathrm{pc})\end{array}$} & \multicolumn{3}{|c|}{$F 435 W$} & \multicolumn{2}{|c|}{$F 814 W$} & \multicolumn{2}{|l|}{ Colors } \\
\hline & & & & $\begin{array}{c}m_{\mathrm{F} 435 \mathrm{~W}} \\
(\mathrm{mag})\end{array}$ & $\begin{array}{c}\text { err } \\
(\mathrm{mag})\end{array}$ & $\begin{array}{c}M_{\mathrm{F} 435 \mathrm{~W}} \\
(\mathrm{mag})\end{array}$ & $\begin{array}{c}m_{\mathrm{F} 814 \mathrm{~W}} \\
(\mathrm{mag})\end{array}$ & $\begin{array}{c}\text { err } \\
(\mathrm{mag})\end{array}$ & $\begin{array}{c}m_{\mathrm{F} 435 \mathrm{~W}}-m_{\mathrm{F} 814 \mathrm{~W}} \\
(\mathrm{mag})\end{array}$ & $\begin{array}{c}\text { err } \\
(\mathrm{mag})\end{array}$ \\
\hline 2035.408 & 810.38 & 11.96 & 6.07 & 25.66 & 0.05 & -9.60 & 24.00 & 0.03 & 1.63 & 0.06 \\
\hline 2032.639 & 745.51 & 49.90 & 25.30 & 25.67 & 0.04 & -9.59 & 25.22 & 0.06 & 0.42 & 0.07 \\
\hline 2036.138 & 823.32 & 9.64 & 4.89 & 25.67 & 0.06 & -9.59 & 24.91 & 0.08 & 0.73 & 0.10 \\
\hline 2034.984 & 821.37 & 4.84 & 2.46 & 25.69 & 0.08 & -9.57 & 24.71 & 0.09 & 0.95 & 0.12 \\
\hline 2034.624 & 830.37 & 12.32 & 6.25 & 25.69 & 0.08 & -9.57 & 24.63 & 0.06 & 1.03 & 0.10 \\
\hline 2035.023 & 817.32 & 6.53 & 3.31 & 25.73 & 0.06 & -9.53 & 24.96 & 0.06 & 0.74 & 0.09 \\
\hline 2036.096 & 816.27 & 10.93 & 5.54 & 25.74 & 0.08 & -9.52 & 24.98 & 0.09 & 0.73 & 0.12 \\
\hline 2034.718 & 827.16 & 9.45 & 4.79 & 25.74 & 0.07 & -9.52 & 24.56 & 0.06 & 1.15 & 0.09 \\
\hline 2035.858 & 817.23 & 7.98 & 4.04 & 25.74 & 0.12 & -9.52 & 25.05 & 0.14 & 0.66 & 0.18 \\
\hline 2035.237 & 813.98 & 8.48 & 4.30 & 25.74 & 0.06 & -9.52 & 24.78 & 0.06 & 0.93 & 0.09 \\
\hline 2034.685 & 827.22 & 9.84 & 4.99 & 25.74 & 0.07 & -9.52 & 25.21 & 0.10 & 0.50 & 0.12 \\
\hline 2034.878 & 815.52 & 9.00 & 4.56 & 25.74 & 0.06 & -9.52 & 26.05 & 0.20 & -0.34 & 0.21 \\
\hline 2035.768 & 823.81 & 5.21 & 2.64 & 25.75 & 0.07 & -9.51 & 24.87 & 0.08 & 0.85 & 0.11 \\
\hline 2035.893 & 814.89 & 9.93 & 5.03 & 25.75 & 0.07 & -9.51 & 24.70 & 0.06 & 1.02 & 0.09 \\
\hline 2034.798 & 824.47 & 7.41 & 3.76 & 25.78 & 0.08 & -9.48 & 25.09 & 0.09 & 0.66 & 0.12 \\
\hline 2034.922 & 816.55 & 7.95 & 4.03 & 25.81 & 0.07 & -9.45 & 25.42 & 0.11 & 0.36 & 0.13 \\
\hline 2035.585 & 829.24 & 7.47 & 3.79 & 25.81 & 0.08 & -9.45 & 25.07 & 0.09 & 0.71 & 0.12 \\
\hline 2035.667 & 824.98 & 4.66 & 2.36 & 25.81 & 0.08 & -9.45 & 25.29 & 0.12 & 0.49 & 0.14 \\
\hline 2034.980 & 826.95 & 6.73 & 3.41 & 25.83 & 0.10 & -9.43 & 24.10 & 0.08 & 1.70 & 0.13 \\
\hline 2034.836 & 828.72 & 9.22 & 4.67 & 25.85 & 0.11 & -9.41 & 24.64 & 0.09 & 1.18 & 0.14 \\
\hline 2035.865 & 814.09 & 10.33 & 5.23 & 25.86 & 0.07 & -9.40 & 24.99 & 0.07 & 0.84 & 0.10 \\
\hline 2035.527 & 832.06 & 9.98 & 5.06 & 25.89 & 0.06 & -9.37 & 25.67 & 0.12 & 0.19 & 0.13 \\
\hline 2036.094 & 817.47 & 10.29 & 5.22 & 25.91 & 0.10 & -9.35 & 24.57 & 0.07 & 1.31 & 0.12 \\
\hline 2036.118 & 818.48 & 10.11 & 5.13 & 25.92 & 0.12 & -9.34 & 24.66 & 0.10 & 1.23 & 0.16 \\
\hline 2035.731 & 813.40 & 10.04 & 5.09 & 25.93 & 0.10 & -9.33 & 24.86 & 0.09 & 1.04 & 0.14 \\
\hline 2034.015 & 837.57 & 22.82 & 11.57 & 25.93 & 0.06 & -9.33 & 23.87 & 0.02 & 2.03 & 0.06 \\
\hline 2034.886 & 824.56 & 6.40 & 3.25 & 25.93 & 0.10 & -9.33 & 25.28 & 0.14 & 0.62 & 0.17 \\
\hline 2034.819 & 826.94 & 8.25 & 4.18 & 25.96 & 0.14 & -9.30 & 25.35 & 0.16 & 0.58 & 0.21 \\
\hline 2035.175 & 830.44 & 8.53 & 4.32 & 25.98 & 0.09 & -9.28 & 25.17 & 0.10 & 0.78 & 0.14 \\
\hline 2034.911 & 822.44 & 5.66 & 2.87 & 25.98 & 0.10 & -9.28 & 24.95 & 0.10 & 1.00 & 0.14 \\
\hline 2035.432 & 814.45 & 7.91 & 4.01 & 25.99 & 0.09 & -9.27 & 25.76 & 0.20 & 0.20 & 0.22 \\
\hline 2034.707 & 821.65 & 8.20 & 4.16 & 26.01 & 0.07 & -9.25 & 25.00 & 0.07 & 0.98 & 0.10 \\
\hline 2035.195 & 832.03 & 10.02 & 5.08 & 26.05 & 0.09 & -9.21 & 25.86 & 0.20 & 0.16 & 0.22 \\
\hline 2033.247 & 752.15 & 39.94 & 20.25 & 26.05 & 0.06 & -9.21 & 24.88 & 0.04 & 1.14 & 0.07 \\
\hline 2034.653 & 834.54 & 15.19 & 7.70 & 26.06 & 0.07 & -9.20 & 25.57 & 0.12 & 0.46 & 0.14 \\
\hline 2034.607 & 821.20 & 9.48 & 4.80 & 26.06 & 0.08 & -9.20 & 24.47 & 0.03 & 1.56 & 0.09 \\
\hline 2035.251 & 818.42 & 4.14 & 2.10 & 26.06 & 0.10 & -9.20 & 24.63 & 0.08 & 1.40 & 0.13 \\
\hline 2035.106 & 817.81 & 5.51 & 2.79 & 26.06 & 0.08 & -9.20 & 25.56 & 0.13 & 0.47 & 0.15 \\
\hline 2035.188 & 832.58 & 10.58 & 5.36 & 26.07 & 0.10 & -9.19 & 24.87 & 0.08 & 1.17 & 0.13 \\
\hline 2034.312 & 87.70 & 19.56 & 9.92 & 26.09 & 0.05 & -9.17 & 25.30 & 0.06 & 0.76 & 0.08 \\
\hline 2034.762 & 821.69 & 7.51 & 3.81 & 26.09 & 0.09 & -9.17 & 25.76 & 0.14 & 0.30 & 0.17 \\
\hline 2034.912 & 818.05 & 7.04 & 3.57 & 26.11 & 0.09 & -9.15 & 25.54 & 0.10 & 0.54 & 0.14 \\
\hline 2034.443 & 817.63 & 12.36 & 6.27 & 26.12 & 0.07 & -9.14 & 25.15 & 0.06 & 0.94 & 0.09 \\
\hline 2034.549 & 824.39 & 10.38 & 5.26 & 26.12 & 0.08 & -9.14 & 24.80 & 0.05 & 1.29 & 0.09 \\
\hline 2035.417 & 831.92 & 9.68 & 4.91 & 26.13 & 0.10 & -9.13 & 25.73 & 0.13 & 0.37 & 0.16 \\
\hline 2035.175 & 812.67 & 9.93 & 5.04 & 26.13 & 0.08 & -9.13 & 25.55 & 0.13 & 0.55 & 0.15 \\
\hline 2034.826 & 834.91 & 14.37 & 7.29 & 26.13 & 0.09 & -9.13 & 25.24 & 0.10 & 0.86 & 0.14 \\
\hline 2035.977 & 814.14 & 11.18 & 5.67 & 26.14 & 0.11 & -9.12 & 25.00 & 0.09 & 1.11 & 0.14 \\
\hline 2033.273 & 756.48 & 36.55 & 18.53 & 26.15 & 0.06 & -9.11 & 25.26 & 0.07 & 0.86 & 0.09 \\
\hline 2033.480 & 84.06 & 29.63 & 15.02 & 26.16 & 0.06 & -9.10 & 25.44 & 0.08 & 0.69 & 0.10 \\
\hline 2035.795 & 822.10 & 5.33 & 2.70 & 26.16 & 0.11 & -9.10 & 25.20 & 0.12 & 0.93 & 0.16 \\
\hline 2036.111 & 817.72 & 10.39 & 5.27 & 26.17 & 0.15 & -9.09 & 24.93 & 0.12 & 1.21 & 0.19 \\
\hline 2034.989 & 835.30 & 13.88 & 7.04 & 26.17 & 0.09 & -9.09 & 25.21 & 0.12 & 0.93 & 0.15 \\
\hline 2035.151 & 819.28 & 4.02 & 2.04 & 26.18 & 0.11 & -9.08 & 24.14 & 0.04 & 2.01 & 0.12 \\
\hline 2035.650 & 816.48 & 6.83 & 3.46 & 26.23 & 0.17 & -9.03 & 25.23 & 0.16 & 0.97 & 0.23 \\
\hline 2034.752 & 821.03 & 7.72 & 3.92 & 26.24 & 0.10 & -9.02 & 25.80 & 0.15 & 0.41 & 0.18 \\
\hline 2033.448 & 84.12 & 29.89 & 15.16 & 26.25 & 0.07 & -9.01 & 25.83 & 0.12 & 0.39 & 0.14 \\
\hline 2035.906 & 815.17 & 9.84 & 4.99 & 26.25 & 0.11 & -9.01 & 25.91 & 0.21 & 0.31 & 0.24 \\
\hline 2034.243 & 815.49 & 15.48 & 7.85 & 26.27 & 0.08 & -8.99 & 25.66 & 0.10 & 0.58 & 0.13 \\
\hline 2035.803 & 89.71 & 13.78 & 6.98 & 26.27 & 0.10 & -8.99 & 25.48 & 0.12 & 0.76 & 0.16 \\
\hline 2035.494 & 811.46 & 10.99 & 5.57 & 26.29 & 0.10 & -8.97 & 25.17 & 0.09 & 1.09 & 0.14 \\
\hline 2034.782 & 828.69 & 9.73 & 4.93 & 26.29 & 0.16 & -8.97 & 24.77 & 0.10 & 1.49 & 0.19 \\
\hline 2035.586 & 829.51 & 7.73 & 3.92 & 26.35 & 0.12 & -8.91 & 25.49 & 0.13 & 0.83 & 0.18 \\
\hline 2036.671 & 817.15 & 17.06 & 8.65 & 26.38 & 0.10 & -8.88 & 25.76 & 0.11 & 0.59 & 0.15 \\
\hline
\end{tabular}


Table 3

(Continued)

\begin{tabular}{|c|c|c|c|c|c|c|c|c|c|c|}
\hline \multirow[b]{2}{*}{$\begin{array}{l}\text { R.A. } \\
\left({ }^{\prime \prime}\right)\end{array}$} & \multirow[b]{2}{*}{$\begin{array}{l}\text { Decl. } \\
\left({ }^{\prime \prime}\right)\end{array}$} & \multirow[b]{2}{*}{$\begin{array}{l}\Delta D^{r m a} \\
(\operatorname{arcsec})\end{array}$} & \multirow[b]{2}{*}{$\begin{array}{c}\Delta D^{r m a} \\
(\mathrm{pc})\end{array}$} & \multicolumn{3}{|c|}{$F 435 W$} & \multicolumn{2}{|c|}{$F 814 W$} & \multicolumn{2}{|l|}{ Colors } \\
\hline & & & & $\begin{array}{c}m_{\mathrm{F} 435 \mathrm{~W}} \\
(\mathrm{mag})\end{array}$ & $\begin{array}{c}\text { err } \\
(\mathrm{mag})\end{array}$ & $\begin{array}{c}M_{\mathrm{F} 435 \mathrm{~W}} \\
(\mathrm{mag})\end{array}$ & $\begin{array}{c}m_{\mathrm{F} 814 \mathrm{~W}} \\
(\mathrm{mag})\end{array}$ & $\begin{array}{l}\text { err } \\
(\mathrm{mag})\end{array}$ & $\begin{array}{c}m_{\mathrm{F} 435 \mathrm{~W}}-m_{\mathrm{F} 814 \mathrm{~W}} \\
(\mathrm{mag})\end{array}$ & $\begin{array}{c}\text { err } \\
(\mathrm{mag})\end{array}$ \\
\hline 2034.588 & 829.18 & 11.94 & 6.05 & 26.38 & 0.12 & -8.88 & 26.10 & 0.22 & 0.25 & 0.25 \\
\hline 2033.347 & 750.35 & 40.55 & 20.56 & 26.41 & 0.09 & -8.85 & 25.41 & 0.08 & 0.97 & 0.12 \\
\hline 2035.611 & 811.63 & 11.15 & 5.65 & 26.42 & 0.11 & -8.84 & 25.63 & 0.14 & 0.76 & 0.18 \\
\hline 2035.145 & 834.46 & 12.54 & 6.36 & 26.45 & 0.12 & -8.81 & 25.39 & 0.12 & 1.03 & 0.17 \\
\hline 2035.110 & 837.33 & 15.43 & 7.83 & 26.47 & 0.12 & -8.79 & 25.12 & 0.07 & 1.32 & 0.14 \\
\hline 2034.626 & 823.86 & 9.35 & 4.74 & 26.50 & 0.12 & -8.76 & 25.89 & 0.16 & 0.58 & 0.20 \\
\hline 2035.505 & 814.93 & 7.59 & 3.85 & 26.51 & 0.15 & -8.75 & 25.59 & 0.16 & 0.89 & 0.22 \\
\hline 2034.683 & 819.32 & 8.98 & 4.55 & 26.51 & 0.14 & -8.75 & 25.96 & 0.18 & 0.52 & 0.23 \\
\hline 2034.756 & 836.28 & 16.02 & 8.12 & 26.60 & 0.11 & -8.66 & 25.61 & 0.11 & 0.96 & 0.16 \\
\hline 2035.326 & 818.40 & 3.93 & 1.99 & 26.62 & 0.23 & -8.64 & 25.79 & 0.39 & 0.80 & 0.45 \\
\hline 2033.440 & 83.67 & 30.24 & 15.33 & 26.62 & 0.11 & -8.64 & 25.80 & 0.11 & 0.79 & 0.16 \\
\hline 2035.306 & 812.33 & 10.00 & 5.07 & 26.65 & 0.15 & -8.61 & 26.00 & 0.20 & 0.62 & 0.25 \\
\hline 2034.941 & 837.09 & 15.79 & 8.01 & 26.67 & 0.13 & -8.59 & 26.44 & 0.21 & 0.20 & 0.25 \\
\hline 2033.077 & 746.71 & 45.47 & 23.05 & 26.68 & 0.12 & -8.58 & 26.27 & 0.18 & 0.38 & 0.22 \\
\hline 2035.968 & 825.06 & 7.98 & 4.05 & 26.71 & 0.14 & -8.55 & 25.66 & 0.13 & 1.02 & 0.19 \\
\hline 2033.964 & 755.00 & 32.36 & 16.41 & 26.71 & 0.11 & -8.55 & 25.57 & 0.08 & 1.11 & 0.14 \\
\hline 2036.043 & 823.36 & 8.47 & 4.29 & 26.73 & 0.14 & -8.53 & 25.44 & 0.12 & 1.26 & 0.18 \\
\hline 2034.281 & 759.78 & 26.21 & 13.29 & 26.82 & 0.13 & -8.44 & 25.51 & 0.08 & 1.28 & 0.15 \\
\hline 2033.286 & 745.82 & 44.62 & 22.62 & 26.84 & 0.11 & -8.42 & 25.86 & 0.11 & 0.95 & 0.16 \\
\hline 2034.191 & 838.22 & 21.72 & 11.01 & 26.86 & 0.15 & -8.40 & 25.54 & 0.09 & 1.29 & 0.17 \\
\hline 2033.781 & 752.17 & 35.93 & 18.22 & 26.87 & 0.12 & -8.39 & 26.12 & 0.14 & 0.72 & 0.18 \\
\hline 2035.355 & 811.94 & 10.40 & 5.27 & 26.90 & 0.15 & -8.36 & 25.75 & 0.14 & 1.12 & 0.20 \\
\hline 2034.992 & 818.45 & 6.02 & 3.05 & 26.94 & 0.19 & -8.32 & 25.34 & 0.10 & 1.57 & 0.22 \\
\hline 2033.034 & 745.66 & 46.60 & 23.63 & 26.94 & 0.13 & -8.32 & 26.10 & 0.14 & 0.81 & 0.19 \\
\hline 2036.127 & 823.85 & 9.57 & 4.85 & 27.02 & 0.21 & -8.24 & 24.81 & 0.06 & 2.18 & 0.22 \\
\hline 2035.138 & 837.69 & 15.72 & 7.97 & 27.03 & 0.19 & -8.23 & 25.43 & 0.09 & 1.57 & 0.21 \\
\hline 2033.365 & 752.69 & 38.59 & 19.56 & 27.15 & 0.16 & -8.11 & 25.80 & 0.12 & 1.32 & 0.20 \\
\hline 2036.659 & 822.99 & 16.06 & 8.14 & 27.15 & 0.22 & -8.11 & 25.43 & 0.11 & 1.69 & 0.25 \\
\hline
\end{tabular}

Note. ${ }^{\text {a }}$ Distance of the star cluster from the position of the $8 \mu \mathrm{m}$ core.

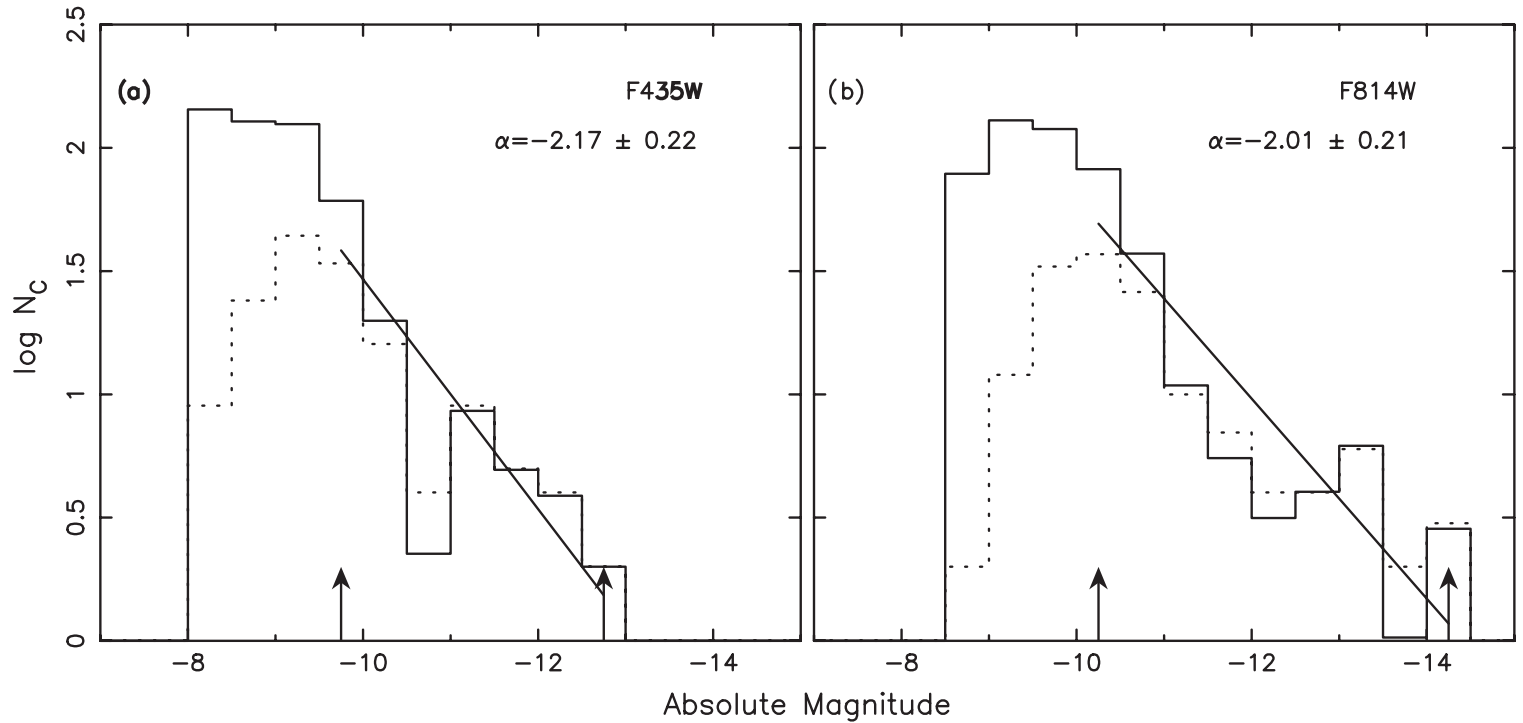

Figure 6. Luminosity function for clusters visible in the $F 435 W$ (a) and $F 814 W$ (b) images of IC 883 . The dashed line histogram is the raw, uncorrected luminosity function, whereas the solid line histogram luminosity function has been corrected for foreground stars and background galaxy contamination, and for completeness. The solid line represents a chi-squared fit to the histogram bins over the magnitude ranges of $M_{\mathrm{F} 435 \mathrm{~W}} \sim-9$ to $-12 \mathrm{mag}$ and $M_{\mathrm{F} 814 \mathrm{~W}} \sim-10$ to $-13 \mathrm{mag}$. The arrows mark the range of the fits, which are done to the $50 \%$ completeness level.

cannot be made between the "B"- and " $P$ "-band-derived values for IC 883 and the $V$-band-derived $\alpha$ values for other wellstudied starburst mergers, it is worth noting that the $\alpha$ values of IC 883 are, within the errors, consistent with the $\alpha$ derived at the $V$ band for NGC $0034(\sim-1.73 \pm 0.1$; Schweizer \& Seitzer
2007) and the Antenna Galaxy ( - 2.13 \pm 0.07 ; Whitmore et al. 2010), but higher than the $\alpha$ of ESO 350-IG038 ( -1.52 \pm 0.05 ; Adamo et al. 2010).

The $F 435 W-F 814 W$ versus $M_{\mathrm{F} 435 \mathrm{~W}}$ color-magnitude diagram is shown in Figure 7 . The approximate range of cluster 


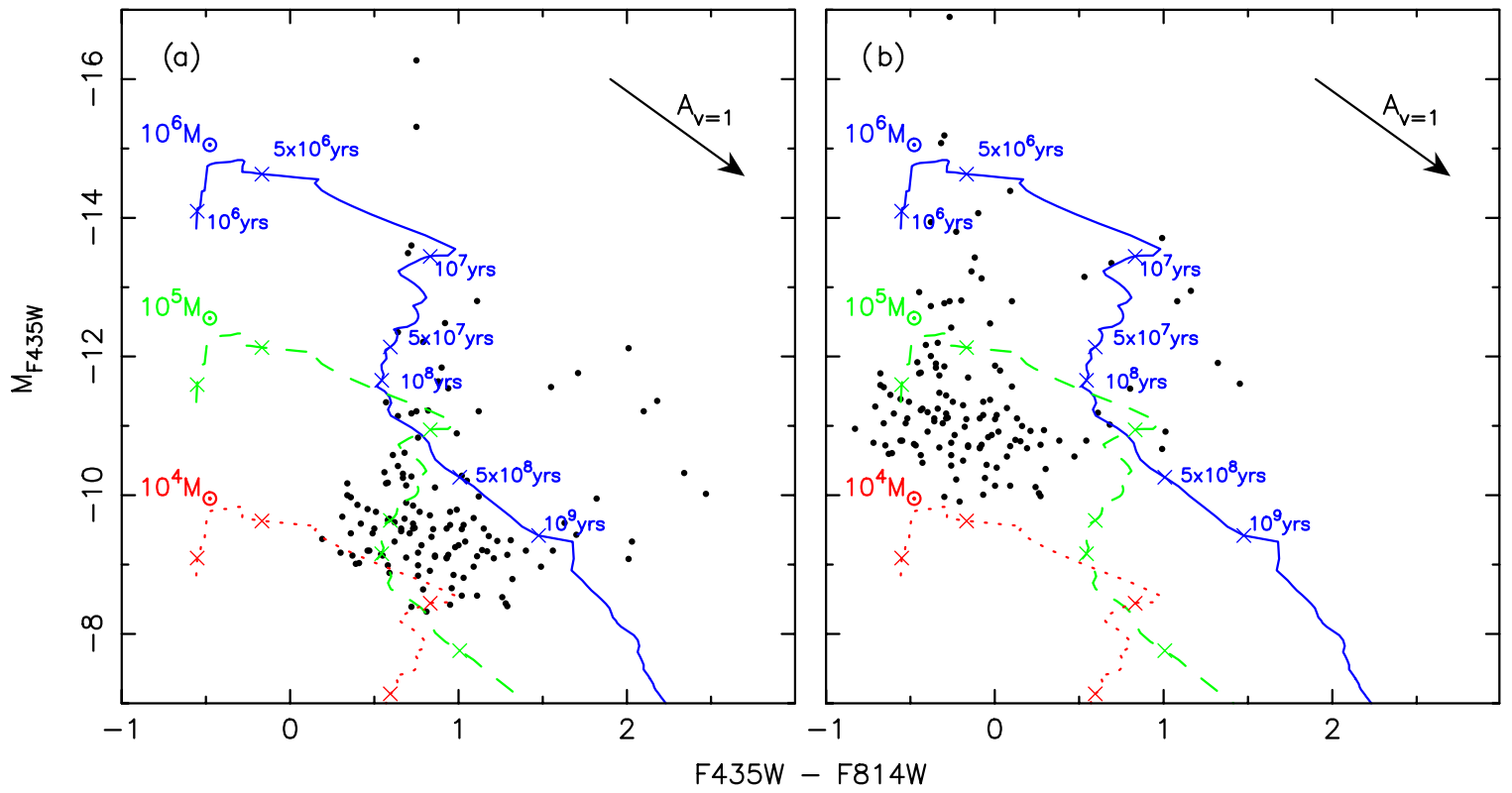

Figure 7. (a) The $F 435 W-F 814 W$ vs. $M_{\mathrm{F} 435 \mathrm{~W}}$ color-magnitude diagram. Bruzual-Charlot population synthesis models with instantaneous starburst and solar metallicity are plotted for cluster masses of $10^{4}, 10^{5}$, and $10^{6} M_{\odot}$. The plotted cluster data have not been corrected for reddening. Only clusters with color uncertainties $<0.2 \mathrm{mag}$ are plotted. The vector represents the magnitude and direction of a cluster in the diagram having $1 \mathrm{mag}$ of visual extinction. For the extinction measurements, the Cardelli et al. (1989) extinction model was used. (b) Same as (a), except the clusters have been corrected for an extinction, $A_{V}=1.2$.

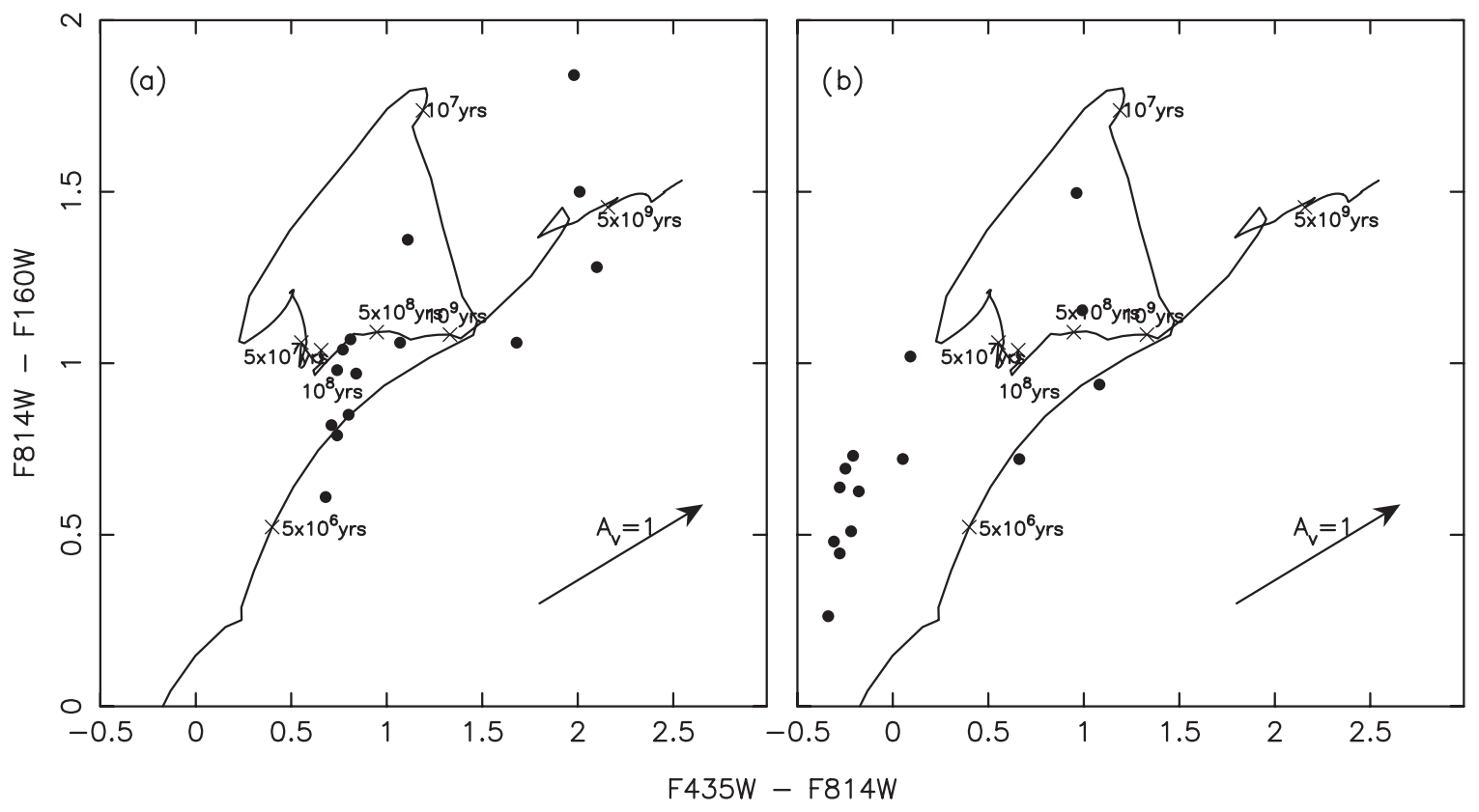

Figure 8. (a) The $F 435 W-F 814 W$ vs. $F 814 W-F 160 W$ color-color diagram. Bruzual Charlot population synthesis models with instantaneous starburst and solar metallicity are plotted. The plotted cluster data have not been corrected for reddening. The color uncertainties of the plotted clusters are $<0.13$ mag. The vector represents the magnitude and direction of a cluster in the diagram having 1 mag of visual extinction. For the extinction measurements, the Cardelli extinction model was used. (b) Same as (a), except the clusters have been corrected for an extinction, $A_{V}=1.2$.

ages is estimated from the data in Figure 7(a) by assuming a Bruzual-Charlot (Bruzual \& Charlot 2003) population synthesis model with an instantaneous starburst, solar metallicity, and a cluster mass of $10^{5} M_{\odot}$ (e.g., see Figure 8 of Surace et al. 1998). Models of clusters with masses of $10^{4}$ and $10^{6} M_{\odot}$ are also shown for comparison. The majority of the clusters lie within the few $\times 10^{7}-10^{8}$ yr age range; there is also an overlap with an age range of $(5-10) \times 10^{6}$ yr for $10^{4} M_{\odot}$ clusters and $>10^{8}$ yr for $10^{6} M_{\odot}$ clusters. By comparison, a similar analysis has been done by making use of archival $1.6 \mu \mathrm{m}(F 160 \mathrm{~W}$ filter) NICMOS data of IC 883 (see Scoville et al. 2000). The ACS-NICMOS color-color diagram $(F 435 W-F 814 W)$ versus $(F 814 \mathrm{~W}-F 160 \mathrm{~W})$, which contains the small number of clusters bright enough to be detectable in the inner $19^{\prime \prime} .5 \times 11^{\prime \prime} .2$ field of view of NIC2 centered on the nucleus, is shown in Figure 8(a). If a synthesis model with identical parameters as that applied to the ACS data is used, the ages for the majority of the clusters are in the range of $\sim(5-500) \times 10^{6} \mathrm{yr}$. These ages 


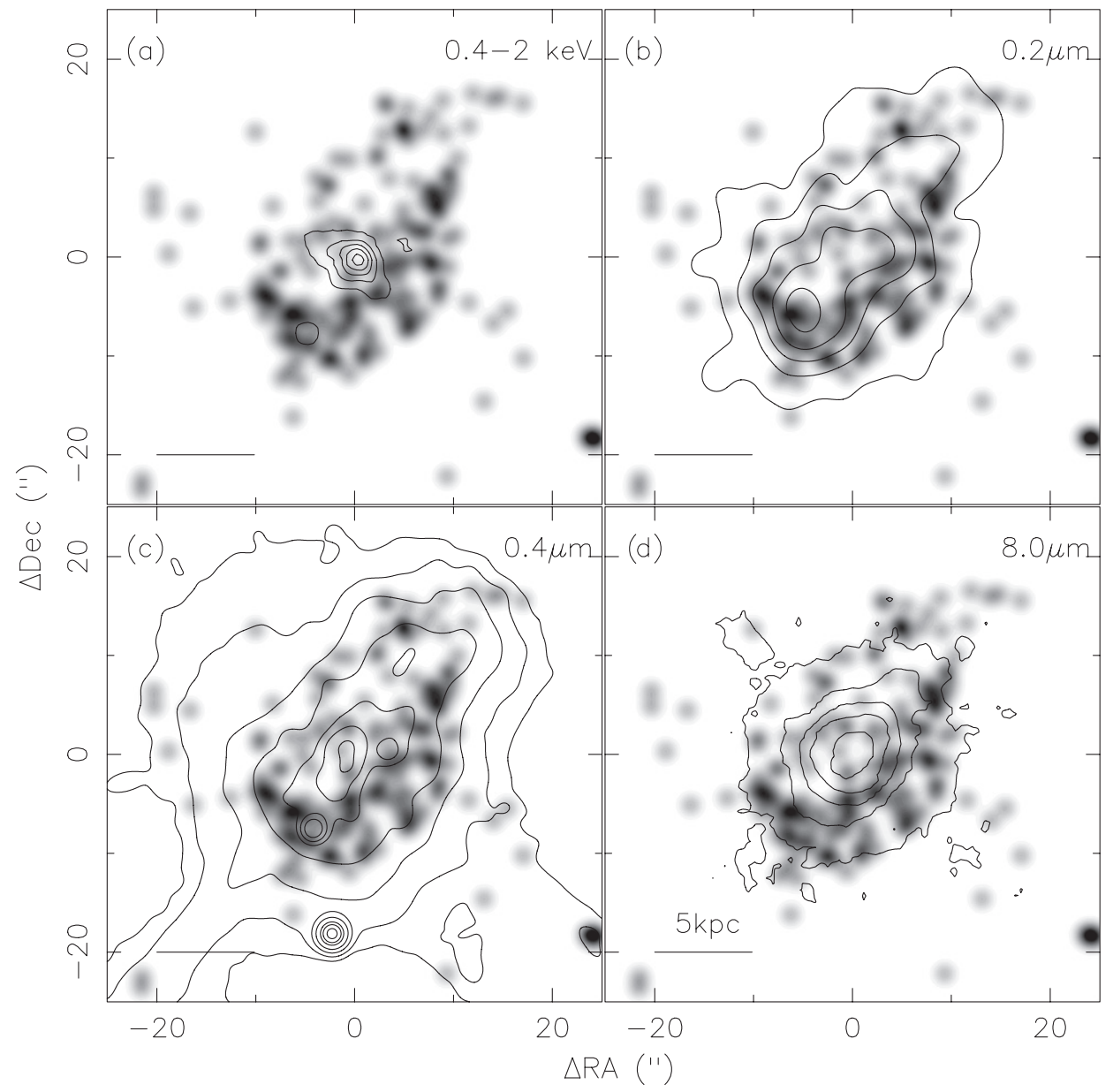

Figure 9. Gray-scale image of star cluster density vs. contours of the (a) Chandra X-ray, (b) GALEX $0.23 \mu \mathrm{m}$, (c) HST $0.4 \mu \mathrm{m}$, and (d) IRAC $8 \mu \mathrm{m}$. In all images, north is up and east is to the left.

are, of course, highly uncertain due to the unknown effects of dust, and the unknown cluster masses, and metallicities of the clusters.

While measurements of extinction in the main body of IC 883 do not exist, Veilleux et al. (1995) made long-slit measurements of the $E(B-V)$ of several LIRGs as part of the original Bright Galaxy Survey. For galaxies with $\mathrm{H} \alpha$ and $\mathrm{H} \beta$ measurements at a nuclear distance $>3 \mathrm{kpc}$-i.e., where the bulk of the clusters in IC 883 reside (e.g., see Section 5.2) - and where the extinction in the long-slit measurements is decreasing with radius, the $E(B-V) \sim 0.4-0.7$. This corresponds to a visual extinction of $A_{V} \sim 1.2-2.2$. This is also consistent with a multi-wavelength analysis of clusters in the starburst galaxy ESO 350-IG038 (Adamo et al. 2010), in which the authors derive extinction values toward the majority of the clusters of $A_{V} \sim 0-2.2$. If an $A_{V}=1.2$ is adopted for the clusters in IC 883, the age estimates (Figure 7(b)) for the bulk of the clusters range from $10^{6}$ to several $\times 10^{7}$ yr for $\sim 10^{5} M_{\odot}$ clusters. In Figure 8(b), such large extinction values do not fit the solar metallicity models for the bulk of the clusters, but are consistent with ages ranging from $10^{6}$ to $10^{8} \mathrm{yr}$ for the reddest clusters.

\subsection{The Distribution of Star Clusters Relative to Emission at Other Wavelengths}

Figure 9 shows the optically visible star cluster density in gray scale relative to the underlying galaxy (in contours) in the soft X-ray band, and at $0.23 \mu \mathrm{m}, 0.44 \mu \mathrm{m}$, and $8 \mu \mathrm{m}$. The star cluster density has been derived by first creating an image containing the position of each star cluster (each with intensity of unity), then applying Gaussian smoothing to the image with a $\sigma=20$ pixels, i.e., the resolution of the $8 \mu \mathrm{m}$ IRAC image. While there are clusters present throughout most of the main body of IC 883 , the highest cluster density regions are located along an arc in the eastern and southern regions of the main body. Clusters are relatively absent in projection toward the optical, $\mathrm{X}$-ray, and $8 \mu \mathrm{m}$ cores. In contrast, the peak of the $0.23 \mu \mathrm{m}$ emission corresponds to the clusters observed in the SE portion of the arc. The offset between the $8 \mu \mathrm{m}$ cores and the cluster arcs is also illustrated in the histogram in Figure 10, which shows the number of clusters as a function of the distance from the $8 \mu \mathrm{m}$ core. The majority of the clusters are at a distance of 3-7 kpc from the core-the peak of the $0.23 \mu \mathrm{m}$ emission corresponds to a distance of $3.2 \mathrm{kpc}$ from the core.

The lack of star clusters observed toward the core of the merger is undoubtedly the result of heavy dust extinction. In Figure 11, the cluster positions are superposed on the $F 814 \mathrm{~W}$ band image of IC 883. Each cluster is coded with a color which represents the $F 435 W-F 814 W$ color bin the cluster belongs to (see the Figure 11 caption). As is clear from the figure, the majority of the reddest clusters lie in or near the reddest nuclear regions of the galaxy. Further support for nuclear reddening comes from the fact that two of the nuclear clusters visible in the $F 814 W$ image are not visible in the $F 435 W$ image, and one of the nuclear clusters visible in the NICMOS $F 160 W$ image is not visible in the $F 435 W$ image. 


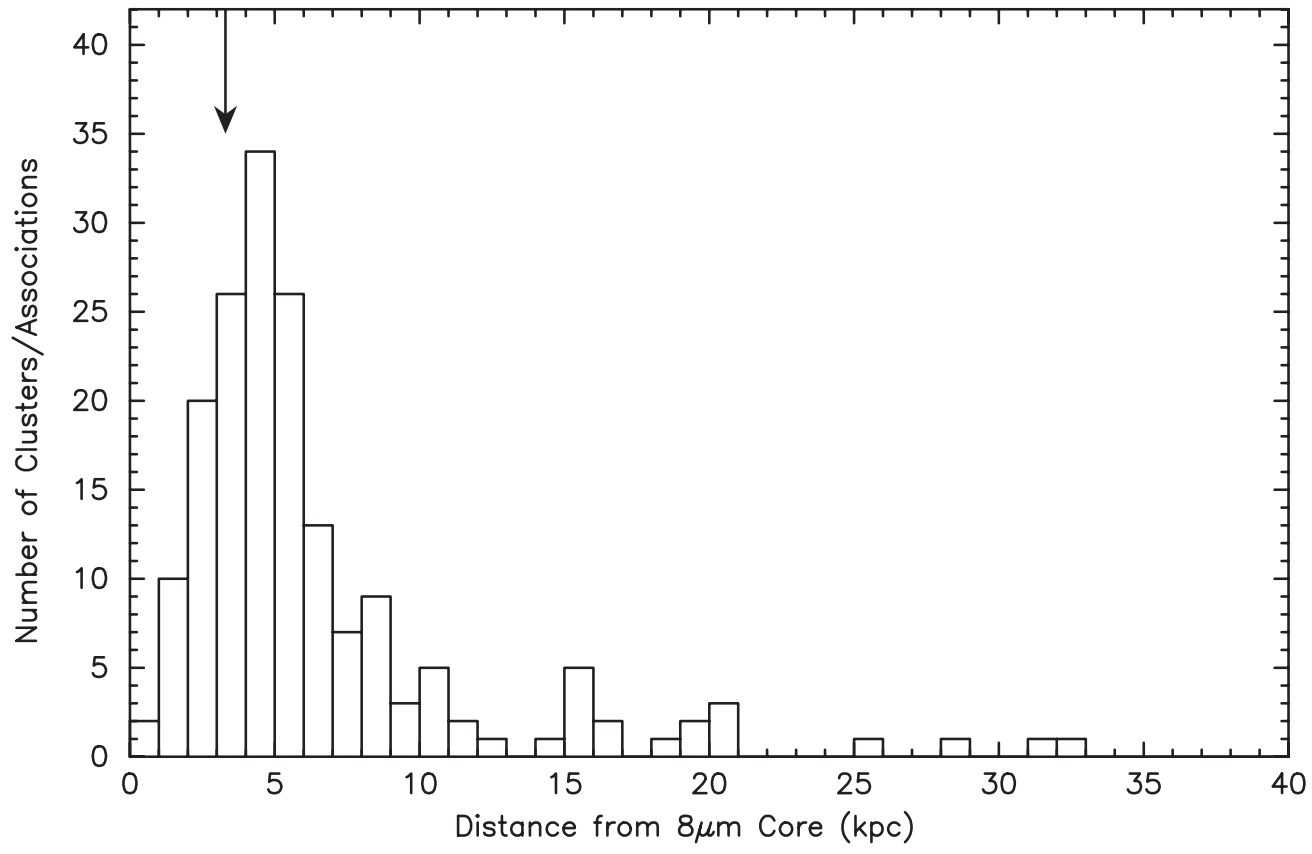

Figure 10. Number of star clusters vs. their distance from the IRAC $8 \mu \mathrm{m}$ core. The position of the GALEX $0.23 \mu \mathrm{m}$ peak is indicated on the plot as an arrow and corresponds to a distance of $3.2 \mathrm{kpc}$ from the $8 \mu \mathrm{m}$ core.

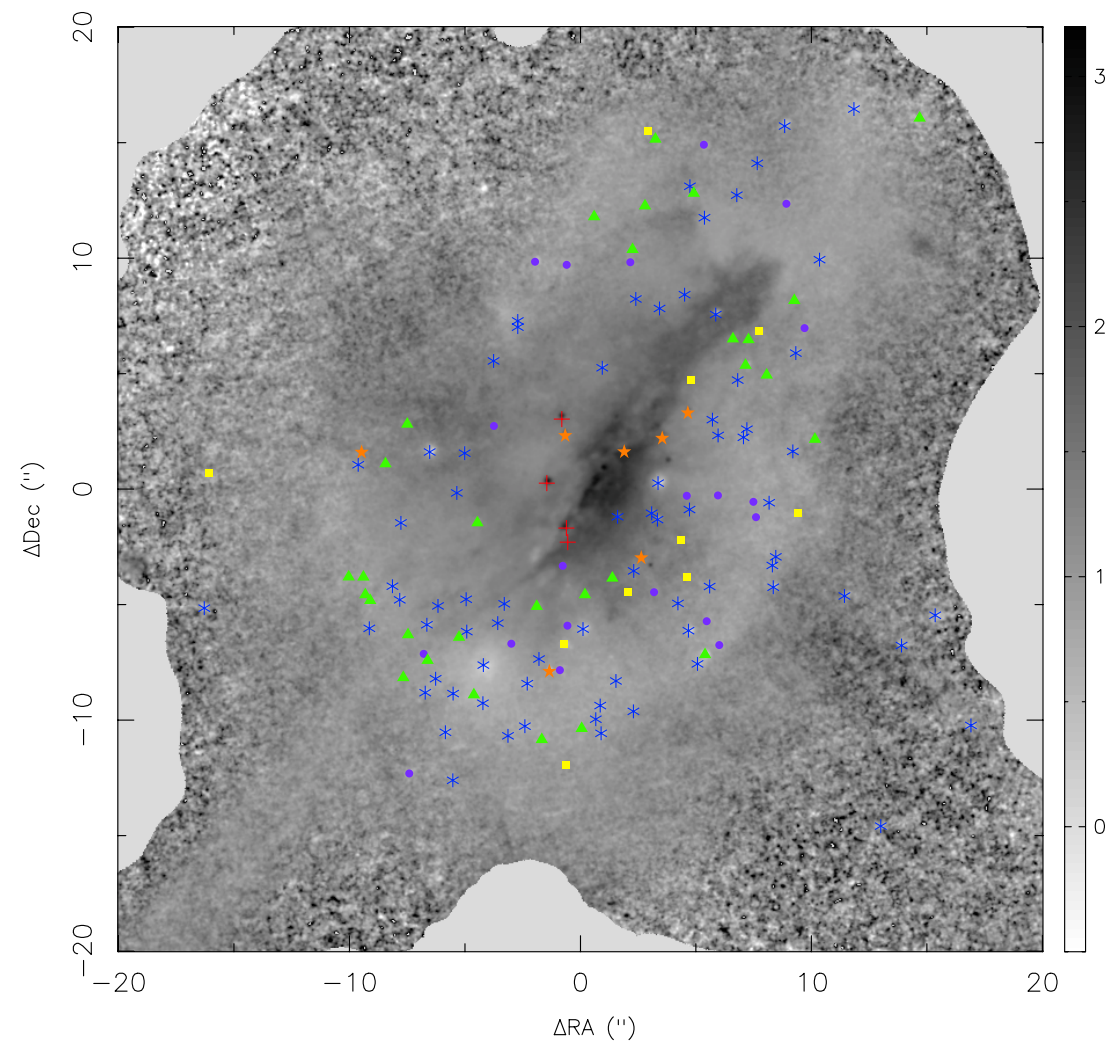

Figure 11. Plot of the position of clusters superposed on an $F 435 W-F 814 W$ gray-scale image of IC 883 . The color value of the cluster represents the $F 435 W-F 814 W$ bin of the cluster. Only clusters with $F 435 W-F 814 W$ mag error less than 0.2 are plotted. The $F 435 W-F 814 W=0-0.5$ bin clusters are designated with violet circles; $F 435 W-F 814 W=0.5-1$ with blue asterisks; $F 435 W-F 814 W=1-1.5$ with green triangles; $F 435 W-F 814 W=1.5-2$ with yellow squares; $F 435 W-F 814 W=2-2.5$ with orange stars; and $F 435 W-F 814 W>2.5$ are designated with red crosses.

The "redness" of the nuclear region is also illustrated in Figures 12(a)-(c), which show the $F 435 W-F 814 W$ grayscale image of IC 883 , with contours of the X-ray, NUV, and $8 \mu \mathrm{m}$ contours superimposed. Figure 12(d) shows the $1.6 \mu \mathrm{m}$ HST NICMOS image in gray scale with the VLA radio, CO, and $8 \mu \mathrm{m}$ contours superposed. The reddest portion of IC 883 clearly lies in a linear strip; in the $1.6 \mu \mathrm{m}$ image, the high surface brightness region of the strip is $\sim 2 \mathrm{kpc}$ across, and the red region in the $F 435 W-F 814 W$ image is $\sim 9 \mathrm{kpc}$ in extent. The core of the primary X-ray emission, the bulk of the mid-IR emission, 


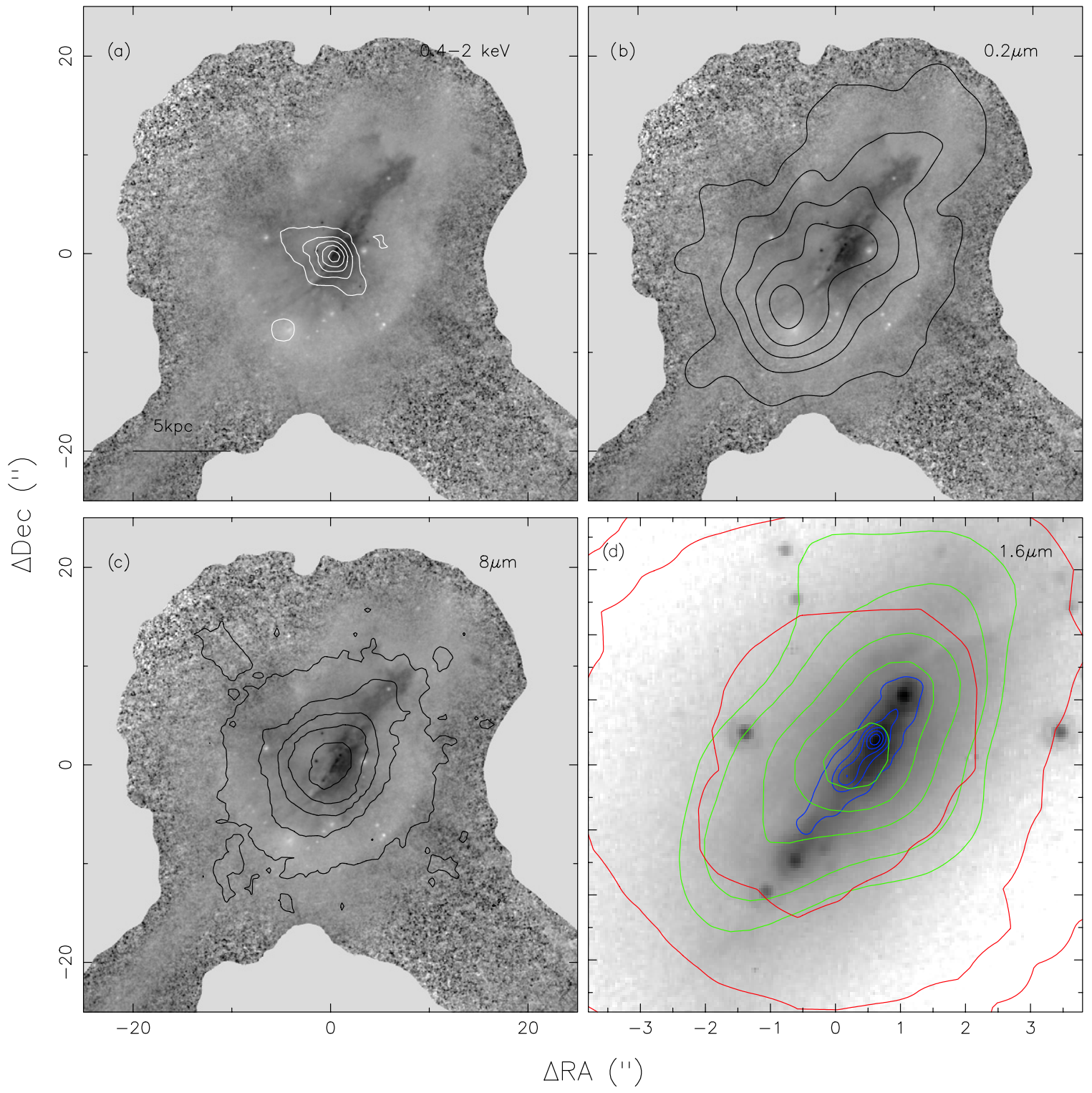

Figure 12. Gray scale of $F 435 W-F 814 W$ vs. contours of (a) Chandra X-ray, (b) GALEX $0.23 \mu \mathrm{m}$, and (c) IRAC $8 \mu \mathrm{m}$. (d) Gray scale $1.6 \mu \mathrm{m} H S T$ NICMOS image with contours of VLA $1.5 \mathrm{GHz}$ (blue), $\mathrm{CO}(1 \rightarrow 0)$ (green), and IRAC $8 \mu \mathrm{m}$ (red) superposed. The $F 435 W-F 814 W$ gray-scale intensity values are the same as those plotted in Figure 11. In all images, north is up and east is to the left.

and all of the VLA- and OVRO-detected radio and CO emission, respectively, lie in these regions.

\subsection{Nuclear Activity and Environment}

Both the Chandra X-ray and Spitzer IRS observations provide probes into the extinguished nuclear regions of IC 883. The hard X-ray emission is extended and the X-ray spectrum (Figure 3) has a hardness ratio which is consistent with X-ray emission from a starburst population. Further, the observed $L_{\mathrm{X}(2-10 \mathrm{keV})} / L_{\mathrm{FIR}(40-120 \mu \mathrm{m})} \sim 8 \times 10^{-5}$ is also consistent with the ratio observed for lower luminosity, $\mathrm{H}$ II-region-dominated galaxies $\left(L_{X} / L_{\mathrm{FIR}} \sim 2 \times 10^{-4}\right.$; Ranalli et al. 2003). Finally, the large equivalent width of the Si XIII line may be due to a contribution of AGN photoionized gas (Section 3; Iwasawa et al. 2011).

The IRS data (Figure 4) provide further support for the $\mathrm{X}$-ray data interpretation of a starburst dominated galaxy with some evidence of an AGN: the low equivalent width of the $6.2 \mu \mathrm{m}$ PAH emission feature $(\sim 0.39 \mu \mathrm{m})$ is consistent with those measured for AGN hosts. Furthermore, the $8 \sigma$ detection of the high ionization [Nev] $14.32 \mu \mathrm{m}$ line is additional evidence that an AGN may be present in IC 883. The $[\mathrm{Ne} \mathrm{v}] /[\mathrm{Ne}$ II] $12.8 \mu \mathrm{m}$ flux ratio of $\sim 0.01$, as well as the low [O IV] $25.9 \mu \mathrm{m} /[\mathrm{Ne}$ II] $12.8 \mu \mathrm{m}$ ratio $(\sim 0.06)$, indicates that the AGN is either extremely weak or that the AGN (and thus the [Ne v]-emitting region) is heavily enshrouded in dust relative to the region(s) where most of the [Ne II] emission is being generated (e.g., see discussion of mid-infrared line diagnostics in Armus et al. 2007 and Veilleux et al. 2009). However, the lack of a strong $9.7 \mu \mathrm{m}$ silicate absorption feature at $10 \mu \mathrm{m}$ rules out any direct evidence of the second possibility.

Is the detection of the $[\mathrm{Ne} \mathrm{v}]$ line truly an indication that an AGN is present? A possible way in which the line might be generated by stellar processes is via supernovae. Consider the supernova remnants (SNRs) 1E0102-72.3 and RCW103: Rho et al. (2009) and Oliva et al. (1999) published a midinfrared spectrum of 1E0102-72.3 and RCW103, respectively, which shows them to have $[\mathrm{NeV}] /[\mathrm{Ne} I I] \sim 0.07$ and 0.01 , 
respectively. Both measurements were made in small apertures $\left(3^{\prime \prime} .7 \times 57^{\prime \prime}\right.$ for $1 \mathrm{E} 0102-72.3$ and $14^{\prime \prime} \times 27^{\prime \prime}$ for RCW103). Thus, if the conservative assumption is made that the $[\mathrm{Ne} \mathrm{v}]$ emission measured in the small apertures is uniformly distributed over the SNRs (i.e., 44" diameter region for 1E0102-72.3 and $105^{\prime \prime} \times 60^{\prime \prime}$ region for RCW103), the [Ne v] luminosities of 1E0102-72.3 and RCW103 are $L_{[\mathrm{Ne} \mathrm{v}]}[\mathrm{SNR}] \sim 5.8 \times 10^{27} \mathrm{~W}$ and $2.5 \times 10^{26} \mathrm{~W}$, respectively. If it is further assumed that these SNRs are representative of SNRs in IC 883 and that the measured [Ne v] flux of IC 883 is its intrinsic flux, approximately $(0.3-7) \times 10^{6}$ SNRs are required to produce the luminosity of the [Ne v] line observed in IC $883\left(L_{[\mathrm{Ne} \mathrm{V}]}[\mathrm{IC} 883]=1.69 \times 10^{33} \mathrm{~W}\right)$. If these $[\mathrm{Ne} \mathrm{v}]$-emitting remnants last $\sim 1000$ years on average, IC 883 would require a steady state production of $(0.3-7) \times 10^{3}$ SNRs $\mathrm{yr}^{-1}$ to sustain the observed [Ne v] luminosity of IC 883. This SNR rate can be compared directly with the rate expected for IC 883 - the star formation rate of IC 883, as measured from either the infrared or radio luminosity and using prescriptions in Kennicutt (1998) and Bell (2003), respectively, is $\dot{M}_{*} \sim$ $80 M_{\odot} \mathrm{yr}^{-1}$. For a Salpeter initial mass function, $\xi(M) \propto M^{-2.35}$ (Salpeter 1955), the number of SNRs produced in IC 883 per year is

$$
\dot{N}_{\mathrm{SNR}} \sim \dot{M}_{*} \frac{\int_{8 M_{\odot}}^{120 M_{\odot}} \xi(M) d M}{\int_{0.5 M_{\odot}}^{120 M_{\odot}} M \xi(M) d M} \sim 1 \mathrm{SNR} \mathrm{yr}^{-1}
$$

Thus, even if the estimated number of SNRs required to produce $L_{\text {[Ne v] }}$ (IC 883) is an order of magnitude off, the required SNR rate would still be too high given the estimated star formation rate.

The nuclear environment in which the nuclear starburst and weak AGNs reside can be assessed with the multi-wavelength imaging data presented in Figure 1. While the Spitzer $70 \mu \mathrm{m}$ MIPS image is of insufficient resolution $\left(\sim 18^{\prime \prime} \sim 9 \mathrm{kpc}\right)$ to constrain the extent of the far-IR emission, the extents of the high surface brightness HST near-IR (Scoville et al. 2000; Figure 12(d)), ground-based mid-IR (Soifer et al. 2001; Figure 1), radio, and $\mathrm{CO}$ emission (e.g., Figure 1) are similar. As a measure of the extent of the far-IR emission, the radio emission will be used as a proxy. This assumption seems reasonable, given that the radio emission is tracing feedback from star formation and/or AGN activity, i.e., processes which can heat their surrounding dust. The extent of the radio emission is $D_{1.4 \mathrm{GHz}} \approx 2.0 \mathrm{kpc}$, and it is inferred from the kinematics of the CO data (Downes \& Solomon 1998) that the CO, and most likely the radio emission (i.e., if the radio emission is associated with star formation), is distributed in a highly inclined disk. Given $D_{1.4 \mathrm{GHz}}$, and the $L_{\mathrm{IR}}$ of IC 883 , the IR surface brightness is estimated to be

$$
\mu_{\mathrm{IR}} \approx \frac{4 L_{\mathrm{IR}}}{\pi D_{1.4 \mathrm{GHz}}^{2}} \approx 2 \times 10^{11} L_{\odot} \mathrm{kpc}^{-2} .
$$

This surface brightness is an order of magnitude less than the theoretical blackbody limit-i.e., if it is assumed that the IR emission is emanating from a spherical blackbody with a luminosity and temperature equivalent to IR luminosity and dust temperature of IC 883, where the dust temperature is estimated from the IRAS 60 and $100 \mu \mathrm{m}$ flux densities, $f_{60 \mu \mathrm{m}}=17.04 \mathrm{Jy}$ and $f_{100 \mu \mathrm{m}}=24.38 \mathrm{Jy}$ (Sanders et al. 2003), via

$$
T_{\text {dust }} \approx-(1+z)\left[\frac{82}{\ln \left(0.3 f_{60 \mu \mathrm{m}} / f_{100 \mu \mathrm{m}}\right)}-0.5\right] \approx 54 \mathrm{~K},
$$

the blackbody size of the IR-emitting region is

$$
D_{\mathrm{BB}}=2\left[\frac{L_{\mathrm{IR}}}{4 \pi \sigma T_{\mathrm{dust}}^{4}}\right]^{0.5} \approx 375 \mathrm{kpc}
$$

where $\sigma$ is the Stefan-Boltzmann constant. The blackbody limit IR surface brightness is thus $5 \times 10^{12} L_{\odot} \mathrm{kpc}^{-2}$. More importantly, the estimated IR surface brightness is less than that of the core of the Orion star-forming region $(\sim 2 \times$ $10^{12} L_{\odot} \mathrm{kpc}^{-2}$ ) and less than that of LIRGs known to harbor luminous AGNs $\left(\sim 10^{13-15} L_{\odot} \mathrm{kpc}^{-2}\right.$; see Soifer et al. 2000 and Evans et al. 2003). Thus, the IR surface brightness and the properties of the radio and $\mathrm{CO}$ emissions are consistent with the above assertion that the IR emission is primarily generated by dust heated by star formation, and that the IR starburst is $2 \mathrm{kpc}$ in extent. As stated above, the estimated star formation rate, calculated from either the IR (Kennicutt 1998) or radio (Bell 2003) luminosity, is $\sim 80 M_{\odot} \mathrm{yr}^{-1}$. This nuclear starburst may be driving the $0.5-2 \mathrm{keV} \mathrm{X}$-ray emission; the morphology of the emission, which extends outward from the nucleus in a direction perpendicular to the radio and CO disk, is suggestive of a stellar wind. Note, however, that this possibility cannot be definitively concluded with the present available data.

\subsection{The Southeastern X-Ray Source}

As stated in Section 3, the X-ray emission has an SE component. This component is coincident with a bright, compact optical knot in the optical images of IC 883. The knot is moderately resolved, with an FWHM extent of $\sim 55$ and 63 pc at $F 435 W$ and $F 814 W$, respectively. The radial profile has a broader shoulder than a Gaussian, with an apparent full extent of $\sim 400 \mathrm{pc}$. Photometrically, it has a $M_{\mathrm{F} 435 \mathrm{~W}}=-16.58 \mathrm{mag}$ and a $F 435 W-F 814 W=0.76 \mathrm{mag}$. Thus, while the SE knot may not be associated with IC 883, the blue color of the knot rules it out as a background source. If the knot has a mass of $\sim 6 \times 10^{6} M_{\odot}$, the estimated age under the assumption that $A_{V}=0$ is 6-7 Myr. The most likely explanations for the nature of the knot are that it is an infalling satellite galaxy, or that it is an off-nuclear star-forming region in IC 883 similar to Knot $\mathrm{S}$ in the Antennae Galaxy (Whitmore et al. 1999). Knot $S$ has $~ 900$ pc diameter; photometrically, it has a $M_{V} \sim-13.81 \mathrm{mag}$, a $B-I \sim 0.39 \mathrm{mag}$, and an estimated age of $7 \pm 1$ Myr. An additional estimation of the stellar age and of the emission line characteristics of the luminous knot via optical Keck spectroscopy will be reported by L.-H. Chien et al. (2012, in preparation).

\subsection{Fueling Extended Star Formation in IC 883}

Finally, an issue worth further investigation is motivated by the similarities in measured fluxes of the interferometric and single-dish measurements of IC 883 . If $20 \%$ calibration errors for all of the measurements are assumed, then the majority (or all) of the $\mathrm{CO}$ emission in IC 883 is traced by the interferometric data, i.e., there is little $\mathrm{CO}$ emission beyond the inner $2 \mathrm{kpc}$ of IC 883. Thus, this small amount of extended emission must fuel the bulk of the optically visible star formation, which accounts for a small fraction of the star formation occurring in IC 883 (i.e., a star formation rate of $0.6 M_{\odot} \mathrm{yr}^{-1}$ based on the far-UV emission; Howell et al. 2010). More sensitive millimeter-wave observations with the IRAM Plateau de Bure Millimeter Array may reveal the molecular clouds associated with the optically luminous regions of star formation observed in the extended regions of IC 883. 


\section{CONCLUSIONS}

Multi-wavelength GOALS observations of the LIRG IC 883 are presented. These observations have provided the most wellrounded view to date of the activity in IC 883. The following conclusions are reached.

1. The luminosity functions of the star clusters have $\alpha \sim$ $-2.17 \pm 0.22$ and $-2.01 \pm 0.21$ at $F 435 W$ and $F 814 W$, respectively. These values are within the range of $V$-band $\alpha$ values measured for the well-studied LIRG NGC 34 and the Antennae Galaxy.

2. The colors and absolute magnitudes of the majority of the star clusters are consistent with instantaneous burst population synthesis models in which the cluster masses are $10^{5} M_{\odot}$ and ages are in the range of a few $\times 10^{7}-10^{8} \mathrm{yr}$. Similar models run by making use of the NICMOS $F 160 W$ data to derive $(F 435 W-F 814 W)$ versus $(F 814 W-$ $F 160 W$ ) yield cluster ages in the range of $\sim(50-100) \times$ $10^{6} \mathrm{yr}$ and $>10^{8} \mathrm{yr}$. If an $A_{V} \sim 1.2$ is adopted, which is consistent with color excesses in LIRGs with measurements of $\mathrm{H} \alpha$ and $\mathrm{H} \beta$ at $>3 \mathrm{kpc}$ and the derived $A_{V}$ values for clusters in ESO 350-IG038, the estimated ages are calculated to be as low as few $\times 10^{6-7} \mathrm{yr}$.

3 . The highest density of optically visible clusters are observed in an arc $\sim 4-5 \mathrm{kpc}$ from the optical and $8 \mu \mathrm{m}$ core. This peak in the optical cluster density also corresponds to the peak in the near-UV emission from IC 883. Both the color of the clusters seen along the line of sight to the nuclear core and the underlying galaxy in the core region are suggestive of heavy nuclear extinction. This is also supported by the fact that the mid- and far-infrared, radio, $\mathrm{CO}$, and $\mathrm{X}$-ray emissions peak in the optically red region.

4. The X-ray hardness ratio and the $L_{\mathrm{X}(2-10 \mathrm{keV})} / L_{\mathrm{FIR}(40-120 \mu \mathrm{m})}$ are both consistent with star formation being the major contributor to the hard nuclear X-ray emission from IC 883. However, the large equivalent width of the Si XIII emission line may be due, in part, to contributions from AGN photoionized gas.

5. The CO, near-IR, and radio emissions have morphologies consistent with the nuclear starburst being situated in an edge-on disk $2 \mathrm{kpc}$ in diameter. An infrared surface brightness $\sim 2 \times 10^{11} L_{\odot} \mathrm{kpc}^{-2}$ is derived under the assumption that the extent of the infrared emission is similar to that of the radio emission. The derived infrared surface brightness is a factor of 10 less than that of the Orion star-forming region.

6. The high ionization [ $\mathrm{Ne} \mathrm{v}] 14.32 \mu \mathrm{m}$ emission line is also detected. The emission is further evidence that an AGN is present-it is determined that the [ $\mathrm{Ne} \mathrm{V}]$ luminosity of IC 883 is too high to be due solely to SNRs. In addition, the low equivalent width of the $6.2 \mu \mathrm{m}$ PAH feature $\sim 0.39 \mu \mathrm{m}$ is also consistent with an AGN. The ratio of [Ne v]/[Ne II] $12.8 \mu \mathrm{m}$ is extremely low at $\sim 0.01$, and the ratio of [O IV] $25.9 \mu \mathrm{m} /[\mathrm{Ne}$ II] $12.8 \mu \mathrm{m} \sim 0.06$. Thus, the AGN appears to be energetically weak relative to the nuclear starburst population.

7. While the bulk of the X-ray emission is coincident with the nucleus, a second X-ray source in IC 883 appears to be associated with a bright, unresolved, off-nuclear component. The component may represent a large starforming region, or an infalling satellite galaxy.
The authors thank G. Soutchkova, L.-H. Chien, A. Leroy, and J. Pizagno for useful discussions and assistance. We also thank the anonymous referee for many suggestions which improved the overall quality and clarity of the manuscript. F.M., T.V., J.P., A.S.E., and D.C.K. were supported by NSF grant AST 02-06262 and by NASA through grants HST-GO10592.01-A and HST-GO11196.01-A from the Space Telescope Science Institute, which is operated by the Association of Universities for Research in Astronomy, Inc., under NASA contract NAS526555. T.V. acknowledges support from the IPAC Fellowship Program. This research has made use of the NASA/IPAC Extragalactic Database (NED) which is operated by the Jet Propulsion Laboratory, California Institute of Technology, under contract with the National Aeronautics and Space Administration. This work is based, in part, on observations made with the NASA Galaxy Evolution Explorer. GALEX is operated for NASA by the California Institute of Technology under NASA contract NAS5-98034. The National Radio Astronomy Observatory is a facility of the National Science Foundation operated under cooperative agreement by Associated Universities, Inc.

\section{REFERENCES}

Adamo, A., Östlin, G., Zackrisson, E., et al. 2010, MNRAS, 407, 870 Armus, L., Charmandaris, V., Bernard-Salas, J., et al. 2007, ApJ, 656, 148

Armus, L., Mazzarella, J. M., Evans, A. S., et al. 2009, PASP, 121, 559

Bell, E. 2003, ApJ, 586, 794

Bertin, E., \& Arnouts, S. 1996, A\&AS, 117, 393

Bruzual, G., \& Charlot, S. 2003, MNRAS, 344, 1000

Bryant, P. M., \& Scoville, N. Z. 1999, AJ, 117, 2632

Cardelli, J. A., Clayton, G. C., \& Mathis, J. S. 1989, ApJ, 345, 245

Condon, J. J., Huang, Z.-P., Yin, Q. F., \& Thuan, T. X. 1991, ApJ, 378, 65

Downes, D., \& Solomon, P. M. 1998, ApJ, 507, 615

Evans, A. S., Becklin, E. E., Scoville, N. Z., et al. 2003, AJ, 125, 2341

Evans, A. S., Mazzarella, J. M., Surace, J. A., et al. 2005, ApJS, 159, 197

Higdon, S. J. U., Devost, D., Higdon, J. L., et al. 2004, PASP, 116, 975

Hinshaw, G., Weiland, J. L., Hill, R. S., et al. 2009, ApJS, 180, 225

Howell, J. H., Armus, L., Mazzarella, J. M., et al. 2010, ApJ, 715, 572

Iwasawa, K., Sanders, D. B., Teng, S. H., et al. 2011, A\&A, 529, 106

Kennicutt, R. C., Jr. 1998, ARA\&A, 36, 189

Kim, D.-C., Sanders, D. B., Veilleux, S., Mazzarella, J. M., \& Soifer, B. T. 1995, ApJS, 98, 129

Mazzarella, J. M., Graham, J. R., Sanders, D. B., \& Djorgovski, S. 1993, ApJ, 409, 170

Mould, J. R., Huchra, J. P., Freedman, W. L., et al. 2000, ApJ, 529, 786

Oliva, E., Moorwood, A. F. M., Draptz, S., et al. 1999, A\&A, 343, 943

Ranalli, P., Comastri, A., \& Setti, G. 2003, A\&A, 399, 39

Rho, J., Reach, W. T., Tappe, A., et al. 2009, ApJ, 700, 579

Rothberg, B., \& Joseph, R. D. 2006, AJ, 131, 185

Salpeter, E. E. 1955, ApJ, 121, 161

Sanders, D. B., Mazzarella, J. M., Kim, D.-C., Surace, J. A., \& Soifer, B. T. 2003, AJ, 126, 1607 (RBGS)

Sanders, D. B., \& Mirabel, I. F. 1996, ARA\&A, 34, 749

Schweizer, F., \& Seitzer, P. 2007, AJ, 133, 2132

Scoville, N. Z., Carlstrom, J. C., Chandler, C. J., et al. 1993, PASP, 105, 1482

Scoville, N. Z., Evans, A. S., Thompson, R., et al. 2000, AJ, 119, 991

Shepherd, M. C., Pearson, T. J., \& Taylor, G. B. 1995, BAAS, 27, 903

Sirianni, M., Jee, M. J., Benítez, N., et al. 2005, PASP, 117, 1049

Soifer, B. T., Neugebauer, G., Matthews, K., et al. 2000, AJ, 119, 509

Soifer, B. T., Neugebauer, G., Matthews, K., et al. 2001, AJ, 122, 1213

Surace, J. A., Sanders, D. B., Vacca, W. D., et al. 1998, ApJ, 492, 116

Van den Bergh, S. 1996, AJ, 112, 2634

Veilleux, S., Kim, D.-C., Sanders, D. B., Mazzarella, J. M., \& Soifer, B. T. 1995, ApJS, 98, 171

Veilleux, S., Rupke, D. S. N., Kim, D.-C., et al. 2009, ApJS, 182, 628

Whitmore, B. C., Chandar, R., Schweizer, F., et al. 2010, AJ, 140, 75

Whitmore, B. C., Zhang, Q., Leitherer, C., et al. 1999, ApJ, 118, 1551 